This document is confidential and is proprietary to the American Chemical Society and its authors. Do not copy or disclose without written permission. If you have received this item in error, notify the sender and delete all copies.

\title{
Exciton-Phonon Interactions Govern Charge-Transfer-State Dynamics in CdSe/CdTe Two-Dimensional Colloidal Heterostructures
}

\begin{tabular}{|c|c|}
\hline Journal: & Journal of the American Chemical Society \\
\hline Manuscript ID & ja-2018-05842x.R2 \\
\hline Manuscript Type: & Article \\
\hline $\begin{array}{r}\text { Date Submitted by the } \\
\text { Author: }\end{array}$ & 04-Sep-2018 \\
\hline Complete List of Authors: & $\begin{array}{l}\text { Pandya, Raj; University of Cambridge Department of Physics, Physics } \\
\text { Chen, Richard; University of Cambridge Department of Physics } \\
\text { Cheminal, Alexandre; University of Cambridge Department of Physics, } \\
\text { Optoelectronics } \\
\text { Dufour, Marion; ESPCI, LPEM } \\
\text { Richter, Johannes; University of Cambridge, Department of Physics } \\
\text { Thomas, Tudor; University of Cambridge Department of Physics, } \\
\text { Ahmed, Shahab; Institute for Manufacturing, Department of Engineering } \\
\text { Sadhanala, Aditya; University of Cambridge, Cavendish Laboratory } \\
\text { Booker, Edward; University of Cambridge Department of Physics, } \\
\text { Department of Physics } \\
\text { Divitini, Giorgio; University of Cambridge, Materials Science and } \\
\text { Metallurgy } \\
\text { Deschler, Felix; University of Cambridge, Cavendish Laboratory } \\
\text { Greenham, Neil; University of Cambridge, Department of Physics } \\
\text { Ithurria, Sandrine; ESPCI, Laboratoire de Physique et d'Etude des } \\
\text { Matériaux } \\
\text { Rao, Akshay; Cavendish Laboratory, University of Cambridge, Physics }\end{array}$ \\
\hline
\end{tabular}




\title{
Exciton-Phonon Interactions Govern Charge-Transfer-State Dynamics in CdSe/CdTe Two-Dimensional Colloidal Heterostructures
}

\author{
${ }^{1}$ Raj Pandya, ${ }^{1}$ Richard Chen, ${ }^{1}$ Alexandre Cheminal, ${ }^{2}$ Marion Dufour, ${ }^{1}$ Johannes M. Richter, ${ }^{1}$ Tudor \\ H.Thomas, ${ }^{3}$ Shahab Ahmed, ${ }^{1}$ Aditya Sadhanala, ${ }^{1}$ Edward P. Booker, ${ }^{4}$ Giorgio Divitini, ${ }^{1}$ Felix \\ Deschler, ${ }^{1}$ Neil C. Greenham, ${ }^{2}$ Sandrine Ithurria and ${ }^{1}$ Akshay Rao*
}

${ }^{1}$ Cavendish Laboratory, University of Cambridge, J.J. Thompson Avenue, CB3 0HE, Cambridge, United Kingdom

${ }^{2}$ LPEM, ESPCI Paris, PSL Research University, CNRS, 10 rue Vauquelin, 75005 Paris, France

${ }^{3}$ Institute for Manufacturing, Department of Engineering, University of Cambridge, 17 Charles Babbage Road, CB3 0FS, Cambridge, United Kingdom

${ }^{4}$ Department of Materials Science and Metallurgy, University of Cambridge, 27 Charles Babbage Road, CB3 0FS, Cambridge, United Kingdom

*ar525@cam.ac.uk

\begin{abstract}
(289 words)
$\mathrm{CdSe} / \mathrm{CdTe}$ core-crown type-II nanoplatelet heterostructures are two-dimensional semiconductors that have attracted interest for use in light-emitting technologies due to their ease of fabrication, outstanding emission yields and tuneable properties. Despite this, the exciton dynamics of these complex materials, and in particular how they are influenced by phonons, is not yet well understood. Here, we use a combination of femtosecond vibrational spectroscopy, temperatureresolved photoluminescence (PL) and temperature-dependent structural measurements to investigate $\mathrm{CdSe} / \mathrm{CdTe}$ nanoplatelets with a thickness of four monolayers. We show that chargetransfer (CT) excitons across the $\mathrm{CdSe} / \mathrm{CdTe}$ interface are formed on two distinct timescales: initially from an ultrafast ( $\sim 70 \mathrm{fs})$ electron transfer and then on longer timescales ( $\sim 5 \mathrm{ps})$ from the diffusion of domain excitons to the interface. We find that the CT excitons are influenced by an interfacial phonon mode at $\sim 120 \mathrm{~cm}^{-1}$ which localizes them to the interface. Using low-temperature photoluminescence (PL) spectroscopy we reveal that this same phonon mode is the dominant mechanism in broadening the CT PL. On cooling to $4 \mathrm{~K}$ the total PL quantum yield reaches close


to unity, with an $\sim 85 \%$ contribution from CT emission and the remainder from an emissive subbandgap state. At room temperature, incomplete diffusion of domain excitons to the interface and scattering between CT excitons and phonons limit the PL quantum yield to $\sim 50 \%$. Our results provide a detailed picture of the nature of exciton-phonon interactions at the interfaces of $2 \mathrm{D}$ heterostructures and explain both the broad shape of the CT PL spectrum and the origin of PL quantum yield losses. Furthermore, they suggest that to maximise the PL quantum yield both improved engineering of the interfacial crystal structure and diffusion of domain excitons to the interface, e.g. by altering the relative core/crown size, are required.

\section{Introduction}

Ultrathin colloidal $\mathrm{CdX}(\mathrm{X}=\mathrm{S}, \mathrm{Se}, \mathrm{Te})$ nanoplatelets are a new class of $2 \mathrm{D}$ materials that have attracted much interest over recent years due to their giant oscillator strength $^{1-3}$, high photoluminescence yields ${ }^{4-7}$ and uniform quantum confinement ${ }^{8,9}$. One characteristic that has however limited the application of these materials in optoelectronic devices, e.g. light-emitting diodes (LEDs) ${ }^{10,11}$ or photocatalytic cells ${ }^{12}$, is the fast non-radiative exciton decay ${ }^{13}$, resulting from the large and exposed surface area ${ }^{14}$. Recently attempts have been made to overcome these limitations through the preparation of heterostructured $\mathrm{CdSe} / \mathrm{CdTe}$ nanoplatelets, consisting of a CdSe nanoplatelet core with a laterally extended CdTe crown (Figure 1) $)^{15}$. These 'heteronanoplatelets', whose thickness can be tuned between 3 - 5 monolayers, has been suggested to have a type-II band alignment where the electron lies in the CdSe core and hole in the CdTe crown $^{3,15,16}$. However, the exact nature of this (type-II vs quasi type-II) is still debated ${ }^{15,16,20}$. The resulting spatial separation between the electron and hole wavefunctions results in (indirect) charge-transfer excitons with a long radiative lifetime ${ }^{3}$. Emission from these CT excitons, formed across the $\mathrm{CdSe} / \mathrm{CdTe}$ interface, is broad (FWHM $\sim 130 \mathrm{meV}$ ) and red-shifted from the domain exciton (excitons in either the CdSe or CdTe domains) absorption by $0.3 \mathrm{eV}$, exhibiting a quantum yield $\sim 50 \%{ }^{15-17}$. Combined with the relative ease of fabrication via solution-processed routes, they are ideal candidates for light-emitting applications as well as model systems for investigating excitons in $2 \mathrm{D}$ heterostructures. 
Several ultrafast spectroscopy studies have been carried out to understand exciton trapping and the multi-exciton states of core-crown $\mathrm{CdSe} / \mathrm{CdTe}$ nanoplatelets ${ }^{18-21}$, which are relevant for LED and lasing applications. These have postulated that, following photoexcitation, domain excitons localize to the interface, to form CT excitons with unit efficiency ${ }^{21}$. These CT excitons are robust to dissociation and can form multi-exciton states with optical gain thresholds in the microjoule per square centimeter regime ${ }^{18}$. However, little has been done to understand how the exciton dynamics, particular of the CT excitons, are influenced by the interfacial structure and phonons. This is a crucial gap in our understanding of these materials because if exciton-phonon interactions are strong it may provide a systematic method for tuning the nanoplatelet emission wavelength and yield by modification of the interfacial crystal structure e.g. via alloying or a gradient composition $^{22,23}$. Furthermore, it could provide insight into the mechanism of exciton localization at the $\mathrm{CdSe} / \mathrm{CdTe}$ interface and explain the broad PL lineshape observed ${ }^{24,25}$. Depending on whether this latter behavior is a result of exciton-phonon interactions or defect states, it may limit device performance. In addition, no studies have addressed the mechanism for PL quantum yield (PLQY) loss. If it is related to the intrinsic non-radiative properties of the CT state, then improved engineering of the interface is likely required. Alternatively, if incomplete diffusion of $\mathrm{CdSe} / \mathrm{CdTe}$ excitons to the interface is responsible, the properties of the pure domains (e.g. size or mobility) should be altered.

Here we combine ultrafast vibrational pump-probe spectroscopy with temperature-dependent steady-state optical spectroscopy and structural measurements to directly interrogate interfacial dynamics in core-crown (CC) nanoplatelets (NPLs) of 4-monolayer (4-ML) thickness. Following photoexcitation of the $\mathrm{CdSe} / \mathrm{CdTe}$ domains we find that CT excitons are formed on two distinct timescales, $\sim 70$ fs and $\sim 5$ ps. The former corresponds to a 'hot' CT population formed from ultrafast electron transfer, and the latter is from the diffusion of domain excitons to the interface. In contrast to previous reports we find that this diffusive transport to the interface does not occur with unit efficiency and, at room temperature, radiative and non-radiative decay of the domain excitons is a major contributor to the PLQY loss at the CT state. On cooling to $4 \mathrm{~K}$ we observe the total PLQY increases to almost unity, with an $85 \%$ contribution from the CT-state emission. At these low temperatures the major loss pathway to CT PL is via emission from a sub-bandgap population. Temperature-dependent PL measurements show that scattering with a phonon mode 
at $\sim 120 \mathrm{~cm}^{-1}$ is responsible for the broad linewidth at room temperature. This same mode, shown via Raman spectroscopy to be present only at the $\mathrm{CdSe} / \mathrm{CdTe}$ interface, is observed in our femtosecond pump-probe measurements to influence the CT exciton dipole moment. We propose that this strong exciton-phonon interaction $(\sim 44 \mathrm{meV})$ acts to localize the CT excitons to the corecrown interface, and at room temperature plays a role in non-radiative losses from the CT state.

\section{Results and Discussion}

$\mathrm{CdSe} / \mathrm{CdTe}$ nanoplatelets, as shown in Figure 1a, were synthesized using a method previously reported $^{15}$. Briefly, cores consisting of 4-monolayer (4-ML) CdSe nanoplatelets were first synthesized (Figure 1a, inset). The core has a zinc blende structure and consists of four Se layers and five Cd layers with a thickness of $\sim 1.5 \mathrm{~nm}$. Laterally around the core, a CdTe crown with the same number of layers was grown by seeded growth. In a flask containing the CdSe core and cadmium precursors, a slow injection of tellurium precursors leads to the lateral extension of the nanoplatelets. Further details of sample preparation and core/crown dimension analysis can be found in the Methods. Unless otherwise stated all measurements were carried out in solution continuously flowed at room temperature on 4-ML nanoplatelets with an overall lateral dimension of $18 \mathrm{~nm} \times 33 \mathrm{~nm}$ (core: 10 x $12 \mathrm{~nm}$; Supporting Information, SI, Figure S1a). Samples were stored, prepared and measured in an inert, oxygen-free environment, to minimize oxidative degradation.

Figure $1 \mathrm{~b}$ shows the absorption (blue) and photoluminescence (red) spectra for 4-ML nanoplatelets in solution. In order to minimize the effects of scattering, measurements were carried out in within an integrating sphere (see Methods). Three strong exciton transitions can be observed in the absorption spectrum: $\sim 45 \mathrm{~nm}(\mathrm{X} 1)$ and $\sim 512 \mathrm{~nm}(\mathrm{X} 2)$ are the electron - light-hole (e-lh) and electron - heavy-hole (e-hh) transitions, respectively, in the CdSe core, whereas the transition at $\sim 555 \mathrm{~nm}(\mathrm{X} 3)$ is the e-hh transition in the CdTe crown ${ }^{15}$. The e-lh transition for the crown appears as a shoulder on the $\mathrm{X} 2$ peak at $\sim 500 \mathrm{~nm}$; these transitions are labelled in Figure 1c. In addition, a further broad tail transition is observed from $590-800 \mathrm{~nm}$. This may be as a result of scattering 
of incoming light from the nanoplatelet potential well ${ }^{20}$ or be the transition to the CT exciton state (with an electron at the CdSe conduction band edge and a hole at the CdTe valence band edge) ${ }^{21}$.

In order to investigate the origin of this absorption feature further and to eliminate the effects of scattering, ultrasensitive absorption measurements were carried out using photothermal deflection spectroscopy (PDS; see Methods) (Figure 1d). The absorption associated with the electronlight/heavy hole transitions can be observed from $2.1-2.8 \mathrm{eV}$. There is also a broad absorption tail from $1.6-2.1 \mathrm{eV}$, the strength of which varies from $1-10 \%$ of the main exciton transitions, as in the linear absorption spectrum (Figure 1c). In $\mathrm{CdSe} / \mathrm{CdTe}$ rods, tetrapods and nanocrystals similar absorption tails have been reported, with the transitions assigned to absorption of the CT state $^{26-28}$. In light of this, and the fact that pure CdSe nanoplatelets do not show such tails in their absorption spectrum $^{1}$ (SI, Figure S2), we deduce that the transitions from $1.6-2.1 \mathrm{eV}$ belong to an energetically broad CT state, as opposed to scattering. Elemental mapping of the nanoplatelets using scanning TEM and energy dispersive X-ray spectroscopy (EDX) ${ }^{15}$ reveals that the $\mathrm{CdSe} / \mathrm{CdTe}$ interface, defined as the region where there is less than $70 \%$ of either CdSe or CdTe, is not sharp. Approximating the lateral width of the interface $\sim 5 \mathrm{~nm}$ gives the oscillator strength of the CT transition to be $\sim 1.5 \%$ of that of the CdTe excitons (SI, Figure S2). For a spatially indirect transition this is a relatively high oscillator strength, especially given the smaller volume of the interfacial region, compared to that of the core/crown (15\% of the crown volume $)^{29,30}$. This can be used to rationalize the relatively large PLQY of core-crown NPLs at room temperature $(\sim 50$ $\%$; see Methods and discussion later) ${ }^{15,17}$. The large oscillator strength will result in a fast radiative decay, which can outcompete non-radiative decay channels, leading to a high CT PLQY (which we define as emission from the CT state between $590-740 \mathrm{~nm}$ ). 
(a)

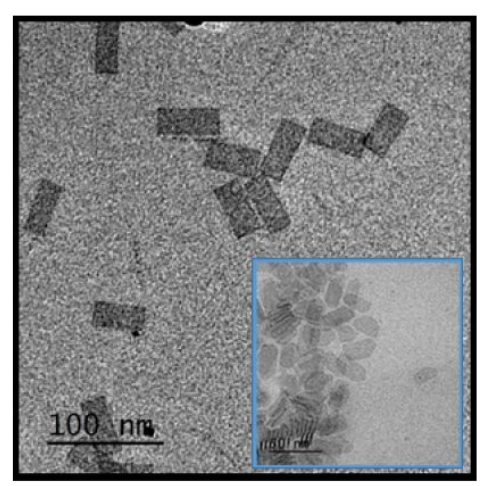

(c)
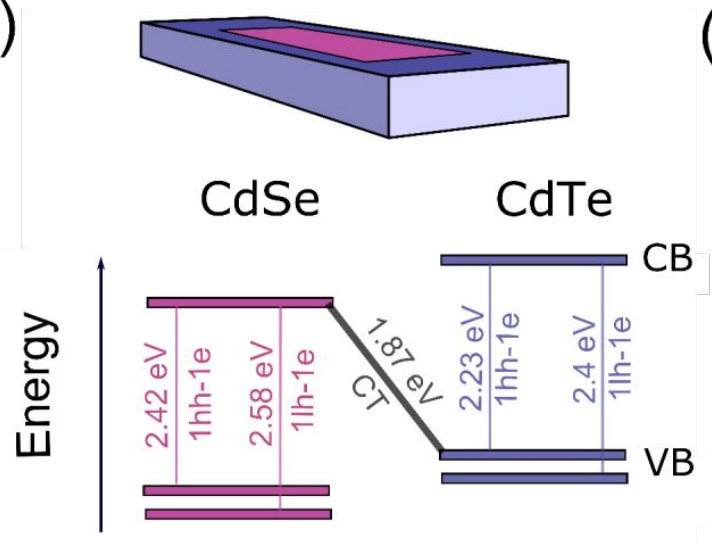

(b)

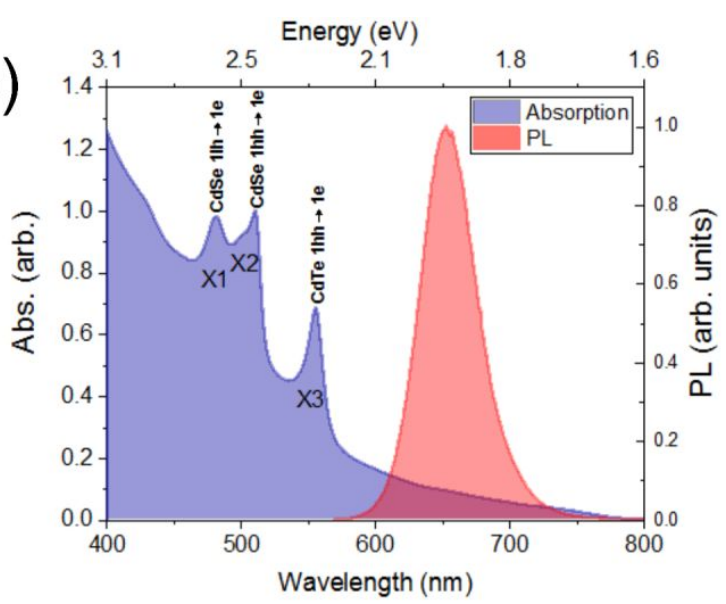

(d)

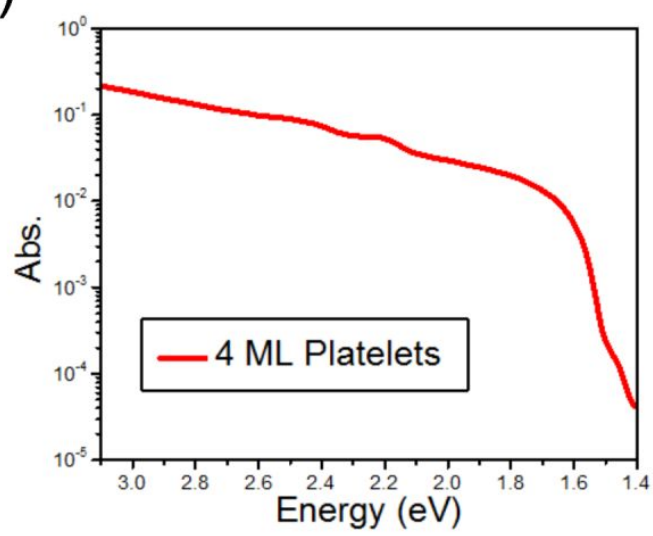

Figure 1: Room-temperature structural and optical properties of 4-ML CdSe/CdTe core/crown nanoplatelets (NPLs). a. TEM images of 4-ML thick CdSe/CdTe core/crown NPLs with a core dimension of $10 \times 12 \mathrm{~nm}$ and an overall dimension of $\sim 18 \times 33 \mathrm{~nm}$. TEM image of CdSe cores is shown in the inset (blue border). b. Absorption (blue) and PL emission (red) spectra for $\mathrm{CdSe} / \mathrm{CdTe}$ core/crown NPLs. The excitonic transitions associated with the CdSe (core) and CdTe (crown) are labelled (X1 - X3). c. Schematic representation of CdSe-CdTe NPLs and associated electronic transitions. d. Photothermal deflection spectrum of 4-ML thick NPLs.

Having identified the origin of the tail transitions, we can use the PDS measurements to quantify the energetic disorder of the nanoplatelets. This is characterized by the Urbach energy, $E_{\mathrm{u}}$, which is related to the absorbance of the material, $A$, by $A(E) \propto e^{E / E u}$, where $E$ is the photon energy ${ }^{31,32}$. Fitting the absorption tail in Figure 1d to the Urbach formula gives an $E_{\mathrm{u}}$ value of $40 \mathrm{meV}$ (SI, Figure S3). This value is approximately four times larger than that obtained for high quality $\mathrm{GaAs}^{33,34}$, but lower than values obtained for $\mathrm{CdS}$ and $\mathrm{CdSe} / \mathrm{CdS}$ nanocrystals $\left(E_{\mathrm{u}} \sim 48-65\right.$ $\mathrm{meV})^{35-37}$ and organic semiconductors ( $\mathrm{P} 3 \mathrm{HT} \sim 50 \mathrm{meV}$ ) ${ }^{38}$. It was observed by Pedetti et al. in the original synthesis of $\mathrm{CdSe} / \mathrm{CdTe}$ nanoplatelets that the inhomogeneous broadening (disorder) contribution to the PL linewidth in these materials is low compared to other type-II nanocrystals ${ }^{15}$. 
This low energetic disorder likely arises from the uniform one-dimensional exciton confinement that can be achieved in nanoplatelets through precise thickness control (see further discussion in SI, Figure S4). 


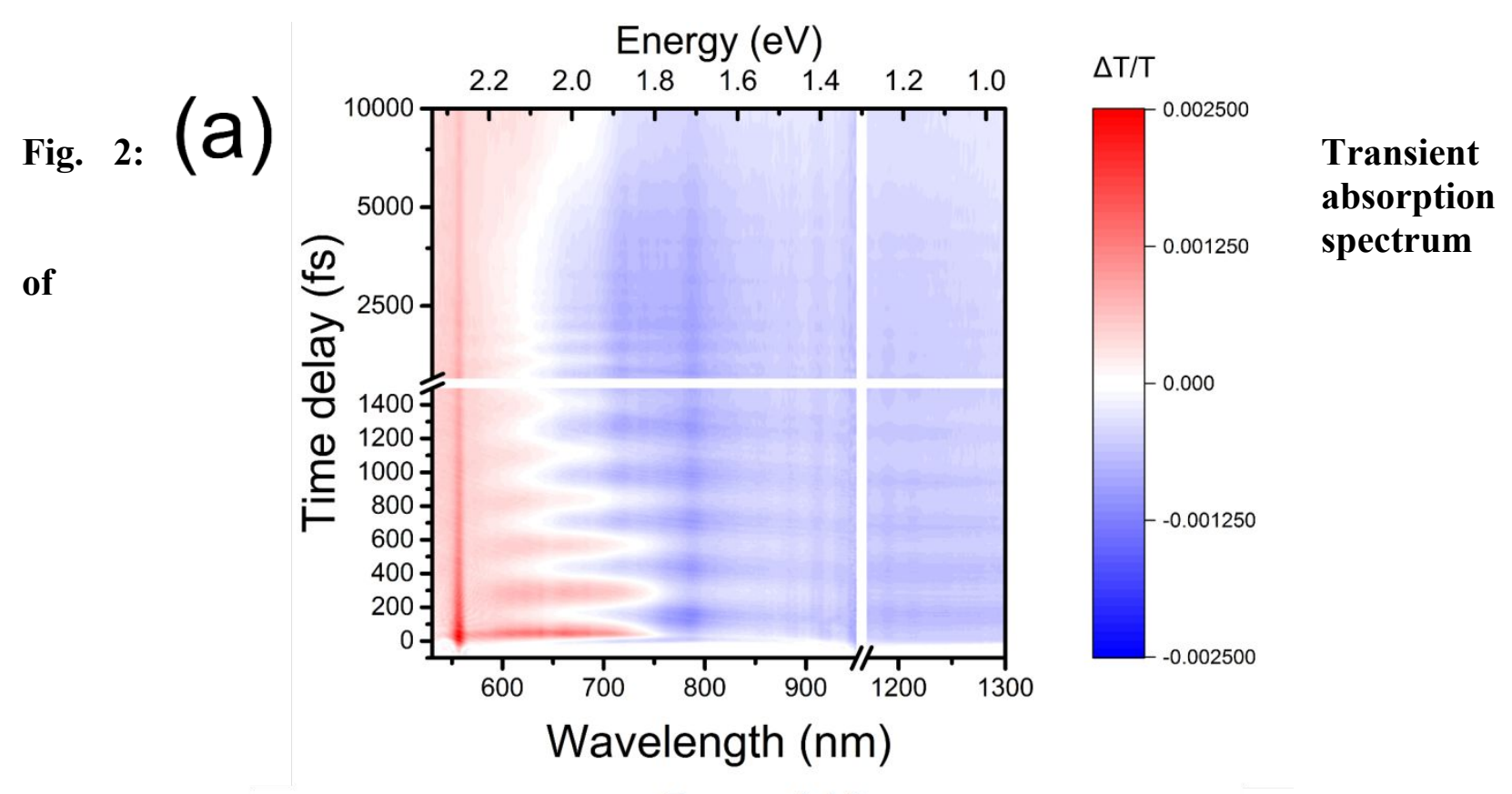

(b)

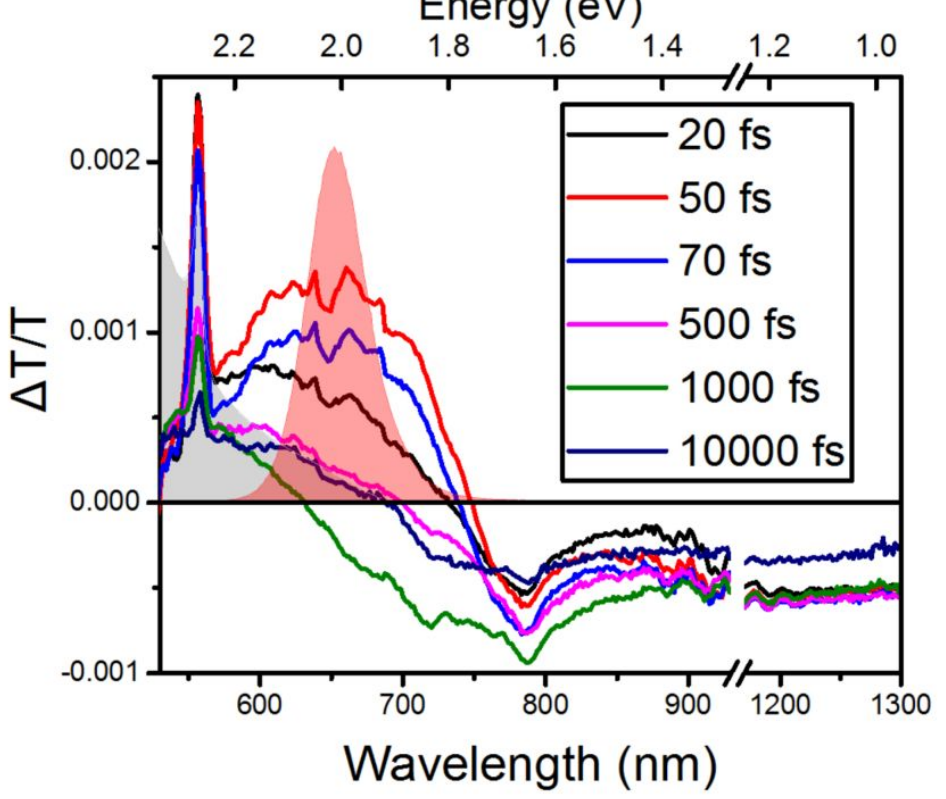

(c)

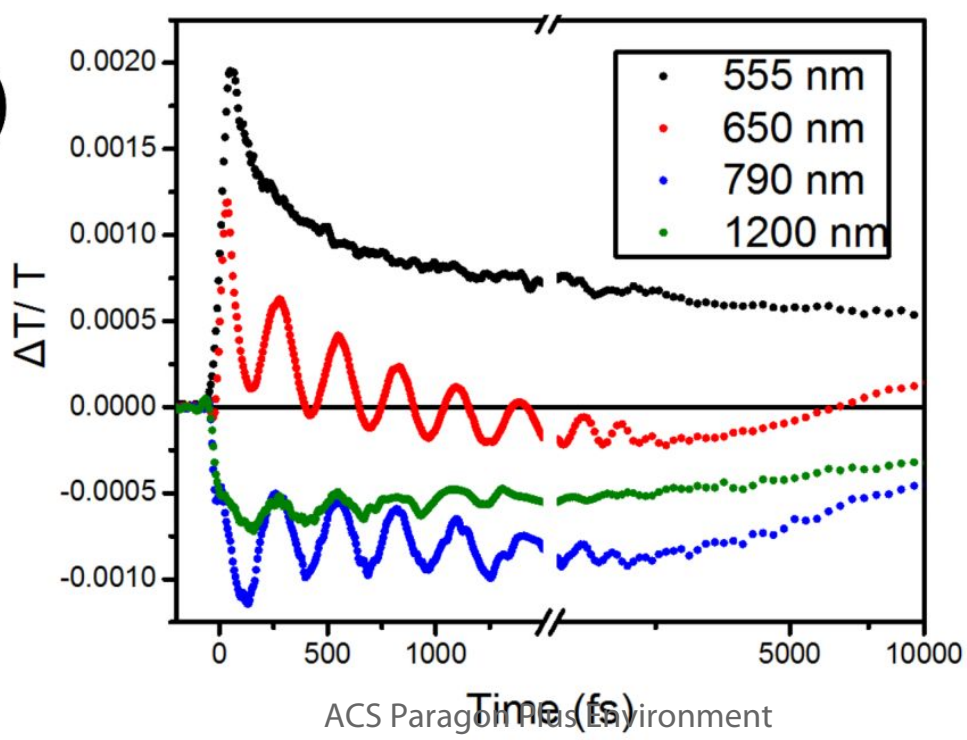


nanoplatelets in solution after excitation with a $\sim 12$ fs pulse centered at $520 \mathrm{~nm}$. a. Differential transmission map of NPLs probing from $530-1350 \mathrm{~nm}$. b. Transient absorption spectra at various pump-probe delays. Grey and red shadings indicate the steady state absorption and emission spectra of core-crown NPLs respectively. The signal around the laser fundamental $1030 \mathrm{~nm}$ is obscured. c. Decay kinetics at various probe wavelengths following photoexcitation.

Although several pump-probe studies have been carried out on $\mathrm{CdSe} / \mathrm{CdTe}$ nanoplatelets ${ }^{18-20}$, no experiments have been able to elucidate how the exciton dynamics are influenced by the interfacial structure. Furthermore, previous studies have focused on the higher-energy $\mathrm{CdSe} / \mathrm{CdTe}$ domain excitons, rather than the CT manifold, whose absorption as shown lies below $2.1 \mathrm{eV}$. In order to directly probe the dynamics of the CT excitons and to understand how these excitons interact with phonon modes, we conducted femtosecond transient absorption (fs-TA) spectroscopy. A solution of nanoplatelets was excited with a $<12$ fs pump pulse (upper limit verified by second-harmonic generation frequency-resolved optical gating (SHG-FROG)) centered at $520 \mathrm{~nm}$ such that it was resonant with the domain exciton transitions. Transitions were probed with a $\sim 300$ fs chirped white light continuum (530-1350 nm). The solution was continuously flowed to prevent laserinduced heating, charge accumulation and sample degradation or aggregation (see Methods). Figure $2 \mathrm{a}$ shows the transient absorption data of 4-ML nanoplatelets, where $\Delta T / T$ is plotted as function of probe wavelength and time delay between pump and probe. $\Delta T$ is the change in the transmission of the sample with/without the pump pulse, and $T$ is the transmission without the pump pulse. In order to determine the lifetimes associated with the various transitions a Fourier filter, tuned to the frequency of the oscillatory component, was first applied to the TA map in order to remove the oscillations which can obscure the fit (SI, S5).

The positive narrow signal centered at around $555 \mathrm{~nm}$ corresponds to the ground state bleach (GSB) of the CdTe e-hh transition, which agrees well with the steady state absorption overlaid in grey shading in Figure $2 b$. This GSB decays with two lifetimes, an initial fast component of $\sim 50$ fs, followed by a much slower decay with a $\sim 22$ ps lifetime. In addition to this, a second positive feature is observed extending from $590-760 \mathrm{~nm}$. This grows in $\sim 65 \mathrm{fs}$, at the same time as the domain exciton GSB decays, and is significantly broader than the CT photoluminescence (red shading; see Figure S5a). The feature at $650 \mathrm{~nm}$ then rapidly decays within $1 \mathrm{ps}$, much shorter than the PL lifetime of $\sim 190 \mathrm{~ns}$ previously reported ${ }^{3,17}$. In ultrafast PL experiments, shown later, no 
emission can be detected in the $590-760 \mathrm{~nm}$ spectral region in the first 10 ps after photoexcitation. Consequently, we assign this feature to a GSB as opposed to any stimulated emission (SE). The poor spectral overlap between the pump pulse centered at $520 \mathrm{~nm}$ (FWHM $\sim 40 \mathrm{~nm}$ ) and the ground-to-CT transition, which has an intrinsically lower oscillator strength than the transitions in the CdSe and CdTe domains, means that the contribution to this bleach from direct CT excitation should be $\sim 100$ times less than the domain exciton GSBs. Therefore, given its magnitude ( $\sim 30 \%$ of the X3 bleach), this GSB can only be the result of an ultrafast electron transfer from the CdTe excitons to a CT state proximal to the interface. CT excitons formed in this manner will have excess energy and may delocalize as they thermalize to the CT band edge ${ }^{20}$. We can hence more specifically assign this broad and rapidly decaying positive feature at early times to the GSB of the 'hot' CT state.

The spectral region $700-970 \mathrm{~nm}$, in Figure $2 \mathrm{~b}$, shows a broad negative feature partially overlapped with the CT GSB. The negative signal indicates that the region corresponds to a photoinduced absorption (PIA), that is, the absorption of excited states generated by the pump pulse. After its initial rise, the PIA continues to grow steadily for around $\sim 2$ ps (blue circles in Figure 2c), matching the decay time of the CT bleach. An exact representation of this PIA is challenging and cannot be accessed from the data presented. However, it appears energetically in the spectrum between PIAs previously assigned to the domain excitons ${ }^{20,21}(>2.1 \mathrm{eV})$ and the PIAs associated with the intraband transitions of free charges, which in nanocrystals are typically below $1 \mathrm{eV}$. In light of this and the fact that the PIA grows in on the same timescale as the decay of the aforementioned 'hot' CT state we assign this feature to 'delocalized CT excitons' i.e. electron-hole pairs that are still correlated but higher in energy than the relaxed CT excitons (Figure S5b). The PIA feature stays roughly constant in magnitude until $\sim 3 \mathrm{ps}$, after which the signal starts to decay slowly with a lifetime of $\sim 36$ ps. At the same time as the PIA begins to decay, we observe a recovery of the bleach at $650 \mathrm{~nm}$. Strong spectral overlap between the CT GSB and PIA prevents definitive assignment of these dynamics. However, the behavior may be a result of diffusion of domain excitons generated away from the interface and the decay of the PIA associated with the delocalized CT excitons. The timescale for this process can be determined by monitoring both the timescale of the recovery and through picosecond transient absorption (ps-TA; Methods and SI, Figure S6) in which the nanoplatelet core and crown are preferentially excited ${ }^{19}$. From this we 


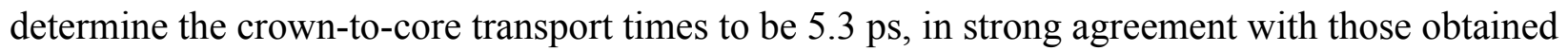
in a recent study by $\mathrm{Li}$ et al. ${ }^{19}$. The $\mathrm{CT}$ population then remains constant to the end of the measurement window (100 ps; SI, Figure S7a), by which point the PIA has become negligible. In the near-infrared region $(1100-1350 \mathrm{~nm})$ a PIA grows in rapidly after photoexcitation and decays with a slightly shorter lifetime $\sim 30$ ps. This could be a PIA associated with the initial domain excitons or the interfacial CT excitons, or both combined. Further experiments are required to elucidate its origin.
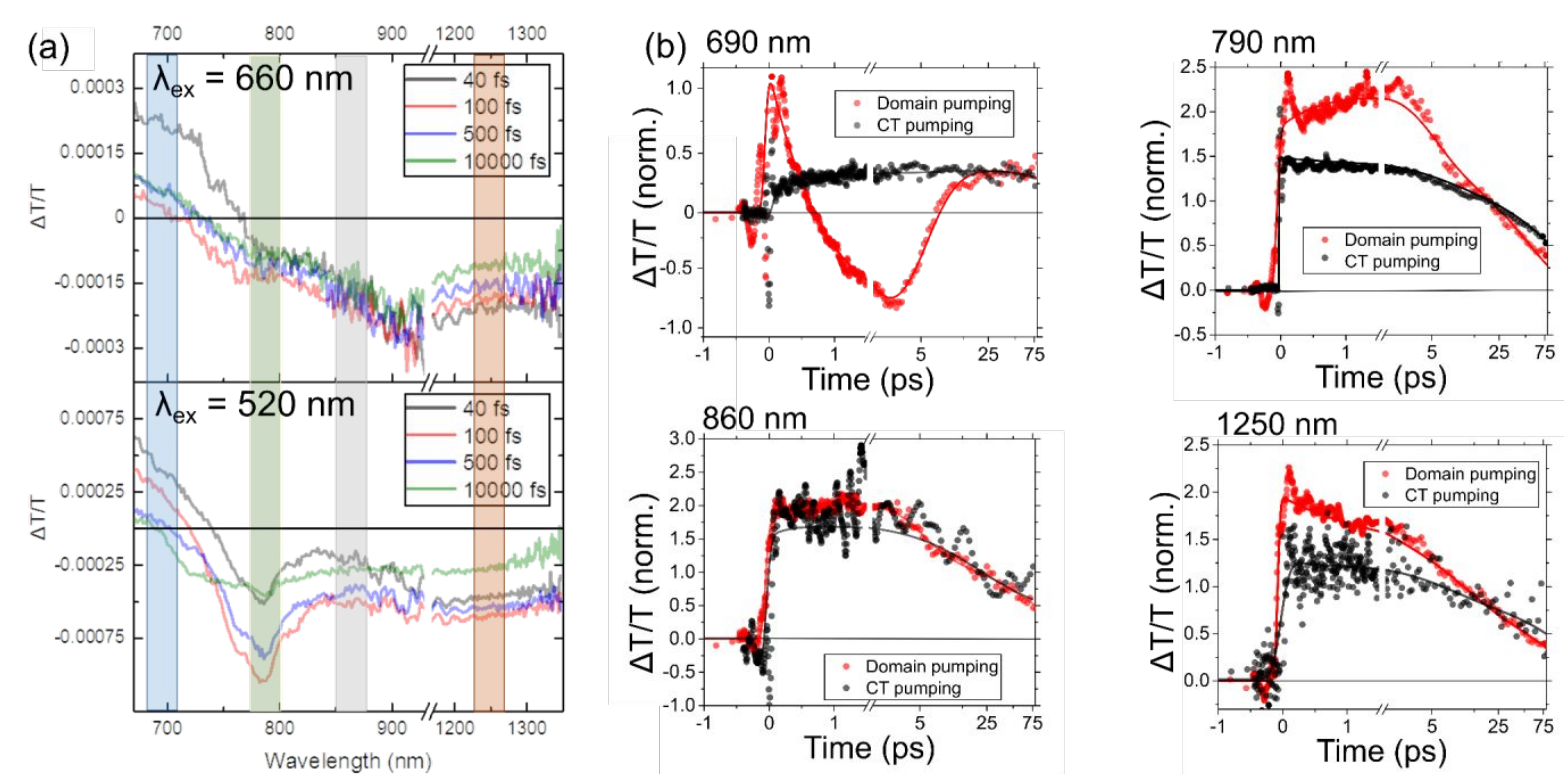

Figure 3: Comparison of electronic response of nanoplatelets following excitation of the core/crown domains and CT state. a. Differential transmission spectrum at various time delays following direct excitation of the CT state at $660 \mathrm{~nm}$ (top) \& domain excitons at $520 \mathrm{~nm}$ (bottom). The shading indicates regions where kinetics in (b) are taken and averaged. b. Comparison of kinetics between CT (black dots) and domain pumping (red dots) at $690 \mathrm{~nm}, 790 \mathrm{~nm}, 860 \mathrm{~nm}$ and $1250 \mathrm{~nm}$. The solid lines are fits to the data. A Fourier filter has been applied to the data for 520 $\mathrm{nm}$ pumping to suppress the oscillatory component and improve the fits. The traces have been normalized to $20 \mathrm{ps}$ to highlight the difference in the early time dynamics.

In order to confirm these observations and further investigate the dynamics of the CT excitons, we tuned our pump wavelength to $660 \mathrm{~nm}$ such as to directly excite the CT state, but not the $\mathrm{CdSe} / \mathrm{CdTe}$ domains. In Figure 3a, we compare the TA spectra for $660 \mathrm{~nm}$ (top) versus $520 \mathrm{~nm}$ (bottom) excitation; the spectra are shown from $680-1350 \mathrm{~nm}$ to exclude effects of pump scatter from the excitation pulse. For both excitation wavelengths the spectral features are similar, with the CT bleach around $690 \mathrm{~nm}$ and a broad PIA extending from $700 \mathrm{~nm}$. However, when pumping 
the CT transitions directly, the bleach at $690 \mathrm{~nm}$ shows a slow rise followed by an almost constant kinetic, instead of the quick initial decay observed when exciting the domain exciton transitions (Figure 3b, top left). Furthermore, the PIA associated with the delocalized CT excitons at 790nm simply decays slowly, instead of growing in the case of domain pumping. This can be rationalized by noting that there is no longer an ultrafast electron transfer to the $\mathrm{CT}$ state as the domain excitons are not excited. Hence no 'hot' CT exciton population is generated. The CT excitons now have much less excess energy to explore the spatial and energetic landscape at the interface and will thermalize to the band edge more rapidly. The sharper features within the PIA at $720 \mathrm{~nm}$ and 790 $\mathrm{nm}$ are not well resolved in the $660 \mathrm{~nm}$ pumping experiment. At longer times this PIA resulting from $660 \mathrm{~nm}$ excitation decays more slowly because the 'delocalized' exciton state is cooler. The PIA in the infrared decays with a similar lifetime of $\sim 30$ ps as observed previously, providing evidence that this PIA is associated with the delocalized CT excitons. 

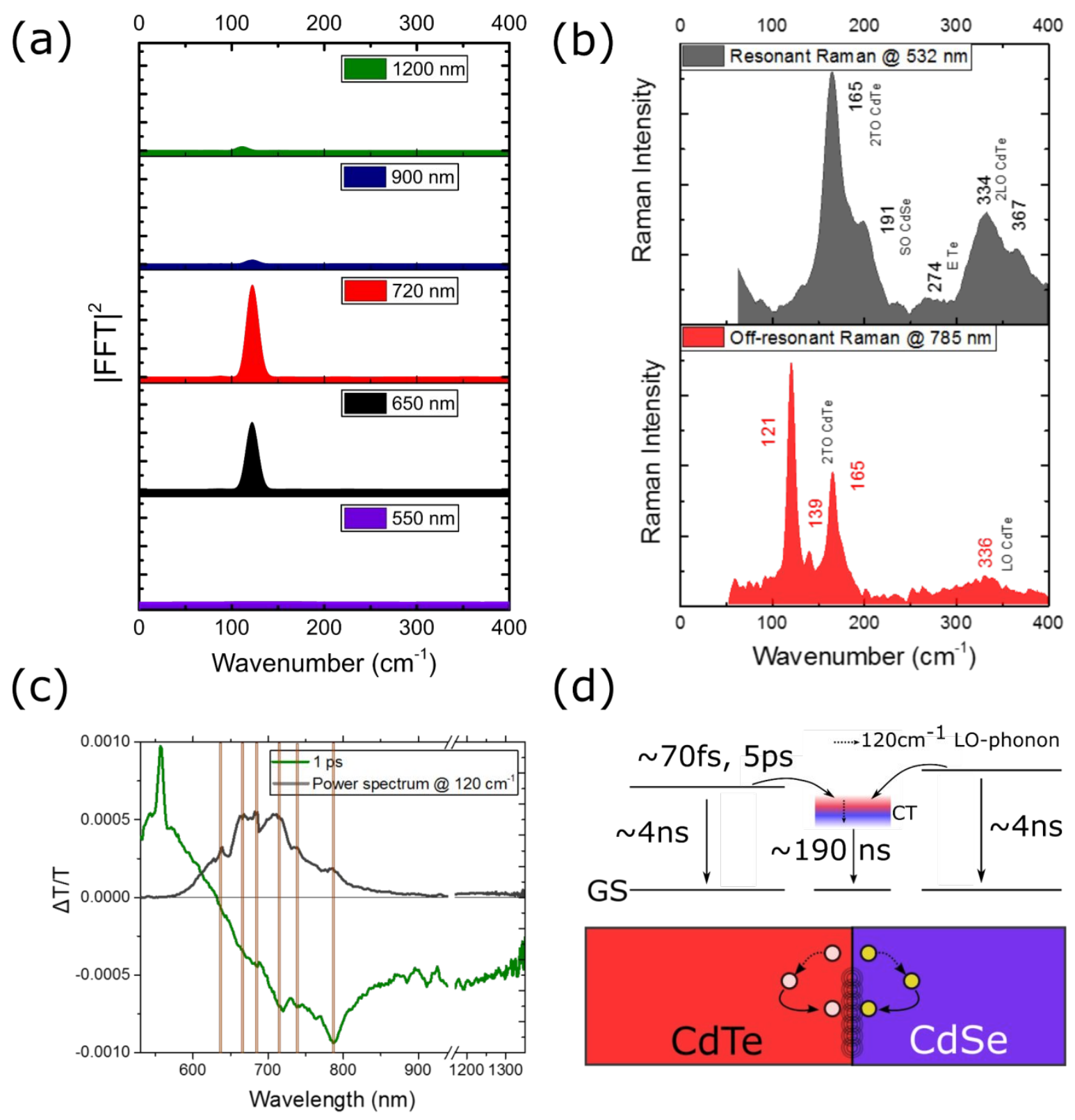

Figure 4: Fourier power spectra and Raman spectra of 4-ML CdSe/CdTe nanoplatelets at room temperature. a. Fourier power spectra at various probe wavelengths. A single mode centered at $\sim 120 \mathrm{~cm}^{-1}$ is observed in the linear time window of measurement $(0.1 \mathrm{ps}-1.5 \mathrm{ps})$. For each spectral slice the intensity of the mode is normalized to the maximum mode intensity across the entire observation window $(530-1350 \mathrm{~nm})$. b. Resonant (532 nm excitation; grey) and offresonant (785 nm excitation; red) Raman spectra. The various Raman modes are marked: 139/165 $\mathrm{cm}^{-1}$ and $330-370 \mathrm{~cm}^{-1}$ modes are TO (transverse optical) and LO (longitudinal optical) phonons of CdTe respectively. The $191 \mathrm{~cm}^{-1}$ peak results from the SO (surface optical) phonons of CdSe and the $274 \mathrm{~cm}^{-1}$ is an E symmetry mode of Te ${ }^{39-42}$. Spectra were collected down to $80 \mathrm{~cm}^{-1}$ due to instrument limitations. c. Intensity of mode at $120 \mathrm{~cm}^{-1}$ as a function of probe wavelength (black line) and TA spectrum at 1 ps (green line). The former is obtained by taking a slice at constant 
frequency through the Fourier transform power map. Orange lines highlight the overlap between the maxima in the power spectrum and the peaks of the PIA. d. Cartoon summarizing energetic processes and associated timescales following photo-excitation of nanoplatelets. CT excitons generated by ultrafast ( $\sim 70 \mathrm{fs})$ electron transfer proximal to the interface possess excess energy (i.e. they are 'hot' CT excitons) as indicated by red shading. They may delocalize at the interfacial region before relaxing through an LO-phonon mode $\left(120 \mathrm{~cm}^{-1}\right)$ to localized 'cold' CT excitons (blue-white shading).

As the samples are excited with a sub-12 fs pump pulse, vibrational wavepackets can be generated on both the ground and excited state potential energy surfaces. These then appear as an oscillatory feature on top of the electronic decay, as seen in the TA map and kinetics in Figure 2. In order to extract the frequency of the vibrational modes, the electronic component of the TA spectrum in Figure 2a was subtracted and the remaining oscillatory component fast Fourier transformed into the frequency domain (see Methods and SI, S7). A single mode centered at $\sim 120 \mathrm{~cm}^{-1}$ (frequency resolution $18 \mathrm{~cm}^{-1}$ ) is observed to modulate the electronic spectrum. In Figure 4a, we plot the Fourier transform spectrum at various probe wavelengths with the mode intensity normalized to the maximum across the map. The mode at $120 \mathrm{~cm}^{-1}$ predominantly modulates the PIA transitions at $790 \mathrm{~nm}$ (and $720 \mathrm{~nm}$ ), previously assigned to the delocalized CT exciton states. There is little to no intensity along the CdTe X3 GSB transition at $555 \mathrm{~nm}$ or in the near-infrared 900-1350 nm. The intensity of this mode at $\sim 650 \mathrm{~nm}$ where the CT GSB resides is also significant. However, examination of the full FFT map and TA spectrum (SI, Figure S8) shows that this is purely a result of the overlap between the CT GSB and the broad PIA. We also note that this same mode is observed to modulate the 700 - $900 \mathrm{~nm}$ PIA transitions of the TA spectrum when directly exciting the CT states of nanoplatelets. This directly proves that the mode is associated with the CT state as opposed to the $\mathrm{CdSe} / \mathrm{CdTe}$ domain excitons. In order to confirm that this phonon mode is indeed modulating an excited state transition, TA measurements were repeated with an off-resonant excitation (17-fs, centered at $860 \mathrm{~nm}$ ), as well as on the blank flow cell and solvent (hexane) (SI, Figure S9a). In either of these cases, no modes are observed (SI, Figure S9). Consequently, we conclude that this mode modulates the excited state of nanoplatelets and more specifically the delocalized CT excitons.

To understand the structural origin of this vibration we can make comparison to resonant (532 nm excitation) and off-resonant (785 nm excitation) Raman spectra, as shown in Figure 4b. 
Comparison with published Raman spectra of CdSe nanoplatelets, CdSe and $\mathrm{CdSe} / \mathrm{CdTe}$ nanocrystals and bulk CdSe/CdTe allows approximate assignment of the various phonon modes ${ }^{39-}$ 42. A few reports on the Raman spectra of CdTe nanocrystals have shown low frequency modes around $120 \mathrm{~cm}^{-1}$ and attributed them to the $\mathrm{A}_{1}$ modes of $\mathrm{TeO}_{2}$ often left as a residual impurity from the synthesis ${ }^{43,44}$. For core-crown nanoplatelets this is unlikely to be the case. Firstly, if this were the case we would expect the mode to modulate the entire electronic spectrum (in both resonant and off-resonant pumping) as opposed to specific transitions. Furthermore, the mode intensity would be strongest around the region of the pump excitation, $\sim 520 \mathrm{~nm}$, where in fact no oscillatory features are observed. Oxidation of nanoplatelets can also be ruled out as samples were prepared, stored and measured in an oxygen-free environment. In addition, elemental analysis of the nanoplatelets using TEM and energy dispersive X-ray spectroscopy (EDX) shows no traces of $\mathrm{TeO}_{2}$ (SI, Figure S1c). Given that the mode is not seen in either CdSe or CdTe individually, we conclude that it is a phonon mode associated with the interface. The presence of this single phonon frequency suggests a high degree of structural order at the interface. Meanwhile, the low frequency of the mode indicates the interface is relatively soft and deformable which will allow strain to be tolerated across the entire nanoplatelet without the quenching of PL by defects, consistent with the high emission yields at room temperature ${ }^{45}$. It should be noted that the phonon mode at $120 \mathrm{~cm}^{-1}$ only appears in the off-resonant Raman spectrum. In resonant Raman the spectrum is dominated by modes associated with transitions directly pumped, i.e. the domain excitons. Modes associated with the transiently generated 'hot' CT state will not have significant population and hence not observable. Taken with the observation that the same mode $120 \mathrm{~cm}^{-1}$ is observed to influence the excited state dynamics when directly pumping the CT state in fs-TA, this underlines the association of this phonon with the interface as opposed to the $\mathrm{CdSe} / \mathrm{CdTe}$ domains. The mode at $120 \mathrm{~cm}^{-1}$ also has a Lorentzian line shape, indicating that it is effectively lifetime-limited and not broadened by disorder ${ }^{46}$; this again suggests that the interface is highly ordered.

Having established that the mode at $120 \mathrm{~cm}^{-1}$ is only present at the interface and specifically influences the delocalized CT excitons, we can interrogate its role in the observed dynamics. In Figure 4c, we plot the intensity of the mode at $120 \mathrm{~cm}^{-1}$ as a function of probe wavelength along with the TA spectrum at 1 ps. The peaks of the wavelength-resolved mode intensity spectrum (orange lines) line up closely with the small peaks within the broad PIA, indicating that the phonon 
mode is modulating the transition dipole moments associated with the PIA spectrum. This causes the TA spectrum to oscillate in intensity over a timescale associated with the mode dephasing time ( $\sim 2$ ps). In other words, the phonon mode is acting to modulate absorption (oscillator strength) of the delocalized CT excitons. This could be because the phonon mode changes the interfacial bond length, i.e. core-crown separation, and hence the electron-hole wavefunction overlap and absorption of the CT state. The mode intensity spectrum (black curve in Figure 4c) effectively represents the absorption of the delocalized $\mathrm{CT}$ exciton state, allowing us to extract this from the overlapping CT GSB. It is well known in semiconductor nanocrystals that interaction with phonons can mediate relaxation ${ }^{47-49}$. In light of this and the evidence presented, we suggest that as they approach the interface, interaction with the phonon mode results in thermalization of the delocalized CT excitons to the band edge. They hence become localized at the interface, contributing to the long-lived CT bleach signal observed after $10 \mathrm{ps}$ in Figure $3 \mathrm{~b}$. This is additional to the previously discussed diffusion of domain excitons to the interface. The timescales for the various dynamical processes discussed are summarized in Figure 4d.

To reconcile our fs-TA measurements with the nanosecond CT photoluminescence lifetimes previously reported ${ }^{15}$, femtosecond transient photoluminescence (fs-PL) measurements were carried out. Solutions of 4-ML nanoplatelets were excited with a $400 \mathrm{~nm}$ pulse where, for the nanoplatelets studied, the crown and core contribute $80 \%$ and $20 \%$ respectively to the total absorption. In order to track the emission on femtosecond timescales we use transient grating photoluminescence spectroscopy ${ }^{50}$ with a time resolution of $\sim 150 \mathrm{fs}$ and collected PL in the range $500-800 \mathrm{~nm}$. As shown in Figure 5a, all emission at early times $(<10 \mathrm{ps})$ is sharp and centered in the $550-580 \mathrm{~nm}$ region. Comparison with steady state PL spectra from the literature shows that this is overlapped with the emission of bare CdTe nanoplatelets. The emission rises on a timescale of $\sim 1$ ps (SI, Figure S10a) suggesting that thermalization of these excitons is relatively slow compared to the $\sim 70$ fs electron transfer to the CT state, as observed in fs-TA. More significantly on this timescale no emission is observed from the interfacial CT states, confirming the positive feature centered at $650 \mathrm{~nm}$ in our fs-TA experiments is not ('cold') CT stimulated emission.

To follow further the fate of excitons generated in the separate $\mathrm{CdSe} / \mathrm{CdTe}$ domains towards interfacial recombination, nanosecond transient photoluminescence (ns-PL) spectra were 
measured using an electronically gated ICCD camera (time resolution $\sim 1.5 \mathrm{~ns}$ ). The spectral map and slices shown in Figure $5 \mathrm{~b}-\mathrm{c}$ (logarithmic scale in Figure S10b) show that there is strong emission from both the CT state, $(\sim 650 \mathrm{~nm})$ and the CdSe core $(\sim 520 \mathrm{~nm})$ and CdTe crown $(\sim 560$ $\mathrm{nm})$. For all three species the rise of the PL is instrument response limited, however the decay of the CT PL can be resolved and is found to decay with three lifetimes of $37 \mathrm{~ns}, 197 \mathrm{~ns}$ and $918 \mathrm{~ns}$ (Figure 5d). In addition to emission from the $\mathrm{CdSe} / \mathrm{CdTe}$ domains and $\mathrm{CT}$ interface there is an additional band, red-shifted with respect to the CT PL centered around $820 \mathrm{~nm}$. Contrary to the CT state emission, this band (purple curve in Figure 5d) shows an initial fast decay $\sim 14 \mathrm{ns,}$ followed by a slower $\sim 286 \mathrm{~ns}$ decay; a third low amplitude decay constant of $1030 \mathrm{~ns}$ is also observed. As will be shown later, emission from this state becomes prominent in the steady-state PL spectrum below $125 \mathrm{~K}$ and can also be accessed by sub-band gap excitation at $647 \mathrm{~nm}$ (SI, Figure S11). The specific origin of this emission is beyond the scope of this work but we note similar emissive species are observed from charged exciton states (trions) in nanocrystals ${ }^{51}$ and pure CdSe nanoplatelets ${ }^{52}$. Alternatively, this emission may arise from the typically lower-energy 'dark' state of the nanoplatelets ${ }^{53}$. 
(a)

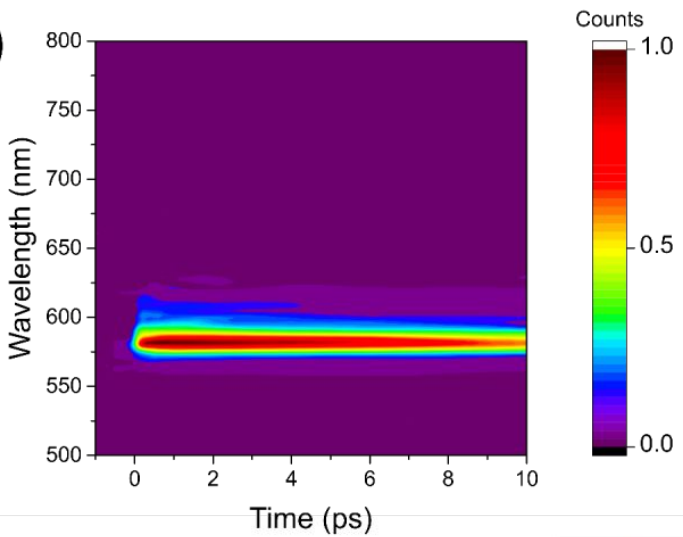

(c)

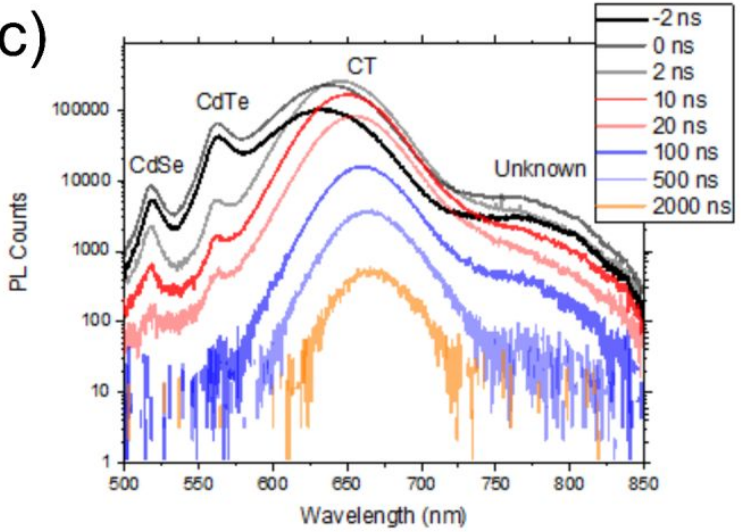

(b)

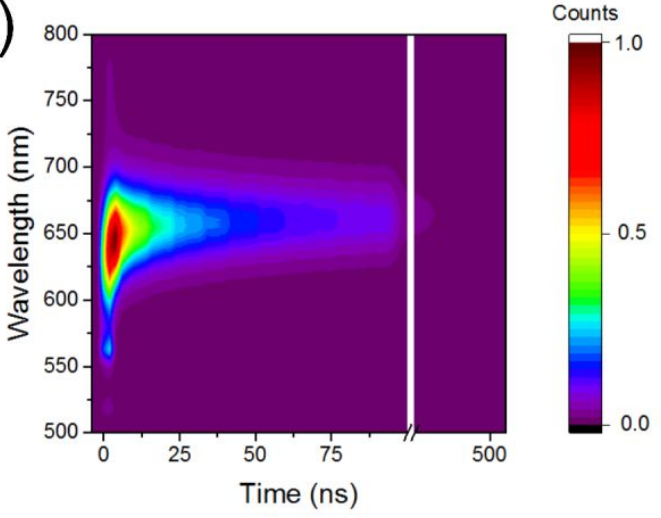

(d)

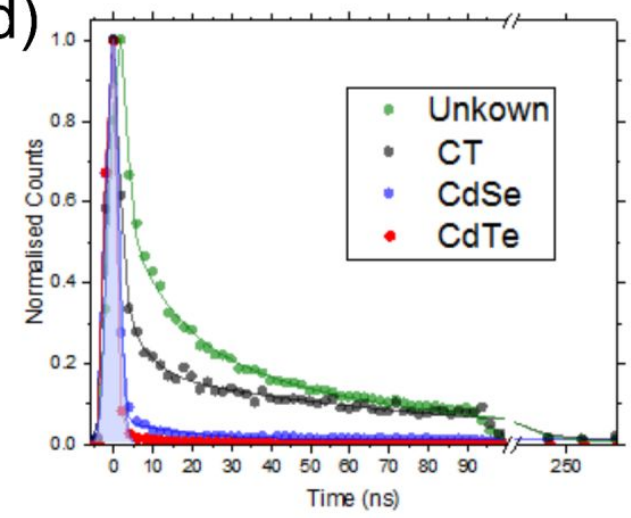

Figure 5: Room temperature transient photoluminescence spectra and kinetics for 4-ML nanoplatelets in solution, with a $400 \mathrm{~nm}$ excitation. a. Femtosecond PL map up to 10 ps with emission collected in the range $500-800 \mathrm{~nm}$. No emission from the CT state is observed around $660 \mathrm{~nm}$. b. Nanosecond PL map shows major emission from CT state and weak emission from domain excitons at early times. c. Spectral cuts at various time delays following photoexcitation in ns-PL experiments. The excitonic emission associated with the CdSe core $(\sim 519 \mathrm{~nm}), \mathrm{CdTe}$ crown $(\sim 562 \mathrm{~nm})$ and CT interface $(\sim 660 \mathrm{~nm})$ are labelled. A fourth hitherto unassigned emissive state $\sim 820 \mathrm{~nm}$ is marked 'Unknown'. d. Decay kinetics associated with the interfacial Unknown, $\mathrm{CT}, \mathrm{CdTe}$ crown and CdSe core exciton recombination. Pale blue shading indicates instrumental response $\sim 1.5 \mathrm{~ns}$.

Given the range of emissive species observed in the transient PL spectrum, it is interesting to ask whether these, as opposed to any non-radiative properties of the CT state, limit the total room temperature PLQY (dominated by CT emission) to $\sim 50 \%$. In core-crown NPLs the presence of an interface introduces new decay channels for the $\mathrm{CdX}(\mathrm{X}=\mathrm{Se}, \mathrm{Te})$ domain excitons. The proportion of excitons that decay via these additional pathways is given by $Q_{C d X}=1-\frac{\left\langle\tau_{C d X, C C}\right\rangle}{\left\langle\tau_{C d X, P u r e}\right\rangle}$ where $\left\langle\tau_{C d X, C C}\right\rangle$ and $\left\langle\tau_{C d X, \text { Pure }}\right\rangle$ denote the average PL lifetimes of CdX excitons in the core-crown (CC) NPLs and pure CdX nanoplatelets of an equivalent structure and composition (see SI, S12 
for further discussion). A fraction, $\alpha$, of the excitons decaying via this alternate pathway will thermalize and reach the band edge, and emit with an efficiency $\eta$. Given that the core $(\mathrm{CdSe})$ and the crown (CdTe) materials have similar zincblende crystal structures and their NPLs have similar PL lifetimes ${ }^{1}$, it is reasonable to assume that $Q_{C d T e}$ is similar to $Q_{C d S e}$ in magnitude. Therefore, the overall PLQY of the nanoplatelet is given by $Q_{C d S e} \cdot \alpha \cdot \eta$. Unfortunately, the decay time of the $\mathrm{CdSe} / \mathrm{CdTe}$ bands cannot be resolved in the experiments in Figure 5. However, using picosecond time correlated single photon counting (TCSPC), as shown in Figure 6a, we determine that $\left\langle\tau_{C d S e, C C}\right\rangle$ and $\left\langle\tau_{C d T e, C C}\right\rangle \sim 0.82 \mathrm{~ns}$ and $\left\langle\tau_{C d S e, \text { Pure }}\right\rangle \sim 4.1 \mathrm{~ns}$, for CdSe NPLs; this results in a value of $\sim 80 \%$ for $Q_{C d S e}$. The challenge of preparing CdTe NPLs of an equivalent composition as in core-crown NPLs means the same analysis is not possible for this domain. The values of $\left\langle\tau_{C d S e, C C}\right\rangle$ and $\left\langle\tau_{C d T e, C C}\right\rangle$ are almost identical likely because similar proportions of their populations decay through the interface. Measuring $\eta$ as $\sim 74 \%$ (PLQY when directly exciting CT state; SI, Figure $\mathrm{S} 12$ ), along with the room temperature PLQY of nanoplatelets $\sim 50 \%$, gives an $\alpha$ of $\sim 79 \%$. Hence for every 100 excitations created in either the core or crown of nanoplatelets, approximately 20 are lost to domain recombination, $\sim 16$ in the thermalization process of domain excitons and $\sim 16$ in non-radiative channels of the cold CT state (this includes emission from the sub-band gap state), leaving 50 for CT emission (Figure 6b). Consequently, to maximize the CT PLQY at room temperature improved engineering of both the domains and interface are required. Reducing the core/crown lateral widths such that the exposed $\mathrm{CdX}$ surface areas are decreased may increase $Q$, as this would result in faster diffusion to the interface. Limiting non-radiative losses from the interfacial region is discussed below.

(a)

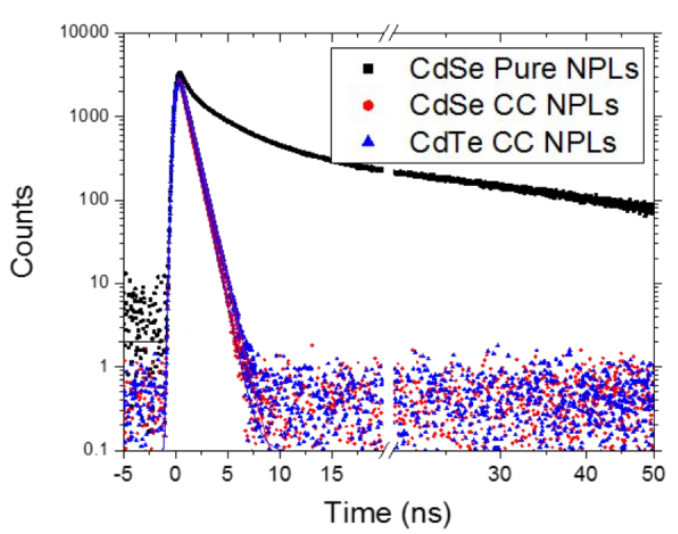

(b)

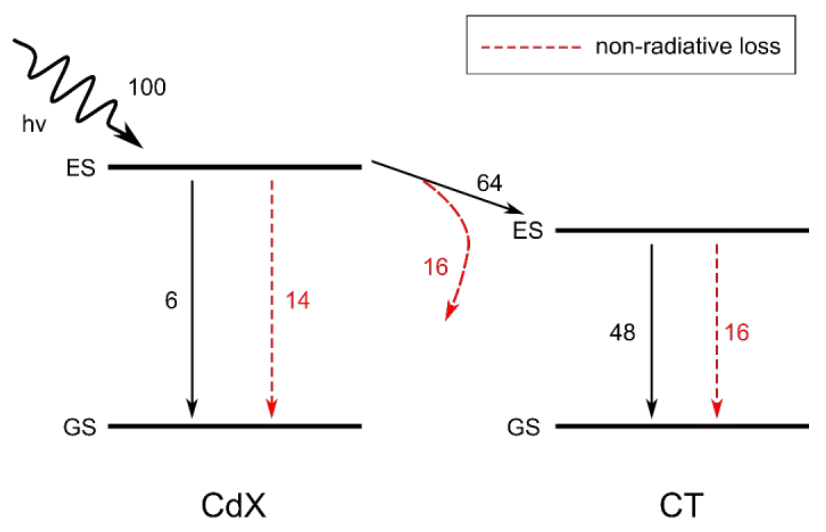


Figure 6: Time correlated single photon counting monitoring $\mathrm{CdX}(\mathrm{X}=\mathrm{Se}$, Te $)$ emission in core-crown (CC) and pure NPLs. a. Decay of CdSe emission in pure 4 -ML NPLs (black), CdSe emission in core-crown NPLs (blue) and CdTe emission in core-crown NPLs (red). Solid lines are fits to data, with decays monitored at 517, 519 and $562 \mathrm{~nm}$ respectively. b. Cartoon summarizing photon loss pathways in nanoplatelets shown for an example input of 100 photons. The black lines indicate photons lost to radiative emission whereas the red dashed lines indicate photons lost nonradiatively. The number of photons emitting radiatively from the $\mathrm{CT}$ state is 48 , approximately equal to the PLQE $\sim 50 \%$.

In order to examine whether inefficient diffusion to and non-radiative decay pathways of the CT state also limit the PLQY at low temperatures, we measured the PLQY of nanoplatelets (excited at $405 \mathrm{~nm}$ ) on cooling to $4 \mathrm{~K}$. Figure 7 a shows the PL spectrum of nanoplatelets as a function of temperature. On cooling there is a concomitant increase in both the total and CT PLQY (Figure 7b; see Methods and SI, Figure S12 for further details). The behavior is almost identical on heating and cooling, i.e. with no hysteresis effect. This is in line with the absence of a phase change, as shown by cryogenic X-ray diffraction (SI, Figure S13). At $4 \mathrm{~K}$ the total PLQY reaches close to unity with that of the CT state emission being $\sim 86 \%$. The steady state spectrum in Figure 7a shows that below $100 \mathrm{~K}$ a new emissive species centered around $820 \mathrm{~nm}$ becomes visible, with a PLQY of $\sim 9 \%$. Hence at low temperatures the major loss pathway competing with CT PL is from the species at $820 \mathrm{~nm}$ previously assigned to a sub-bandgap population. The increase in PLQY on lowering temperature is unusual for nanoparticle systems ${ }^{54}$. When most nanocrystals are cooled, thermalization occurs to the energetically lowest exciton sub-level, which is typically a weakly emitting 'dark' state. This in turn reduces the PLQY. The observation of the opposite behavior warrants further examination, e.g. using magneto-optical techniques, on the exciton fine structure of core-crown nanoplatelets.

To understand whether or not impurities are responsible for the PL quenching as temperature rises, we fitted the wavelength-integrated PL of the CT emission in Figure 7a using a modified Arrhenius formula to estimate $E_{\mathrm{b}}$, the activation energy of the non-radiative decay pathway due to impurities.

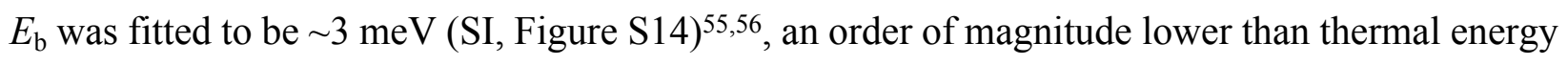
at room temperature suggesting impurities could easily play a significant role in CT PL quenching in nanoplatelets and therefore in limiting the room temperature PLQY. However, as we will show later, the broadening of the CT PL emission cannot be explained merely by impurities with this 
activation energy, but can be explained by the $120 \mathrm{~cm}^{-1}$ phonon mode observed in our fs-TA experiment. It is also noted in Figure $7 \mathrm{~b}$ that there is a sudden drop in the PLQY from $\sim 80 \%$ to $\sim 65 \%$ as the temperature rises above $\sim 150 \mathrm{~K}$; approximately the temperature where the phonon mode at $120 \mathrm{~cm}^{-1}(\equiv 167 \mathrm{~K})$ begins to become populated significantly. This suggests that, at room temperature, scattering with this phonon mode is responsible for the non-radiative losses from the CT state, as opposed to defect quenching. At higher temperatures a larger fraction of excitons are scattered outside the light cone (i.e. to some dark states) as they thermalize to the CT band edge within the interfacial region. They may recombine non-radiatively or relax back into the light cone and emit after a certain time, thereby increasing the CT luminescence lifetime and lowering the overall PLQY. As the temperature drops the phonon scattering mode is essentially shut off and the 'hot' CT excitons thermalize more efficiently to the CT band edge, leading to an increase in the PLQY on cooling; the lower-energy emissive state however prevents the CT PLQY from reaching unity.

(a)

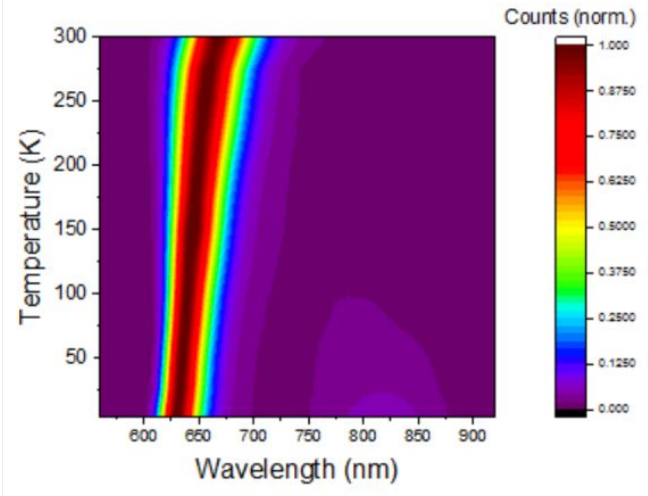

(c)

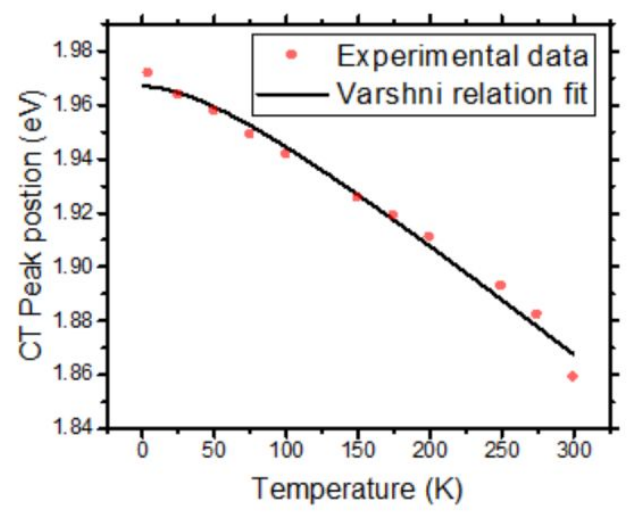

(b)

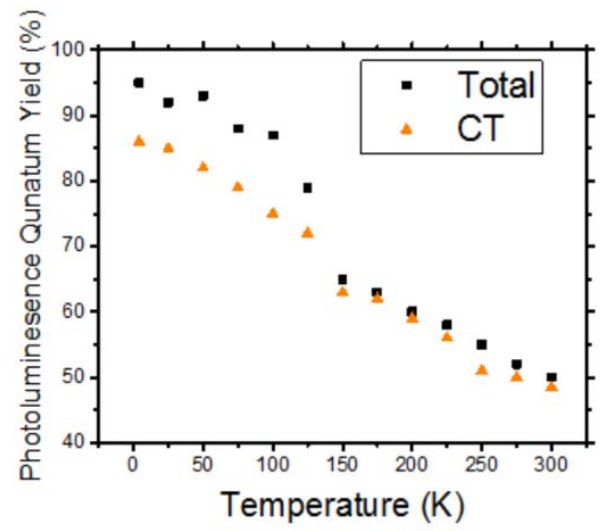

(d)

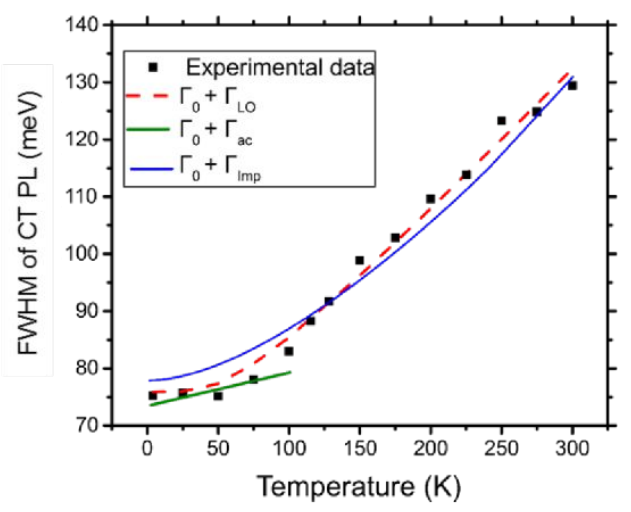


Figure 7: Temperature-dependent photoluminescence of a dispersed film of nanoplatelets excited at $405 \mathrm{~nm}$. a. Temperature-dependent PL map of CdSe/CdTe NPLs obtained on cooling from room temperature. Spectra were recorded in $\sim 25 \mathrm{~K}$ steps with interpolation between temperatures. b. PLQY of core-crown NPLs obtained on cooling down to $4 \mathrm{~K}$. The behavior is approximately identical on heating from $4 \mathrm{~K}$ to room temperature. The black squares indicate the PLQY obtained by integration over the entire spectral region measured $(500-900 \mathrm{~nm})$, whereas the orange triangles represent the PLQY obtained via integration of only the CT band $(590-738$ $\mathrm{nm})$. c. PL peak positions as a function of temperature. The solid line is the best fitted curve with the Varshni relation (Equation 1). d. FWHM of the CT PL as a function of temperature and fitting with various broadening mechanisms. The green line is the combination of inhomogeneous broadening and an acoustic phonon. The red curve shows the effect of inhomogeneous broadening and a $\mathrm{LO}$ phonon $\left(\mathrm{E}_{\mathrm{LO}}=14.8 \mathrm{meV}\right)$ that couples to excitons. The blue dashed curve is the fitting of inhomogeneous broadening and impurity $\left(\mathrm{E}_{\mathrm{b}}=3 \mathrm{meV}\right)$ scattering.

As nanoplatelets are cooled there is also a blue shift of $\sim 0.1 \mathrm{eV}$ in the $\mathrm{CT}$ emission. To understand the origin of this we fit the PL maximum as a function of temperature to the Varshni relation ${ }^{57}$, which is well established in describing the temperature dependence of the band gap in nanocrystals:

$$
E_{g}=E_{0}-\frac{\alpha T^{2}}{T+\beta}(\text { Equation 1) }
$$

In this equation $\alpha$ is the temperature coefficient, $\beta$ is approximately the Debye temperature of the material, and $E_{0}$ is the band gap at $0 \mathrm{~K}$. The results of the fit are shown in Figure $7 \mathrm{c}$ where we obtain values of $\mathrm{E}_{0}=1.967 \mathrm{eV}, \alpha=4.1 \times 10^{-4} \mathrm{meV} / \mathrm{K}$ and $\beta=92 \mathrm{~K}$. Comparing these with the values obtained for bulk CdSe and CdTe from the literature ${ }^{58-61}\left[\mathrm{CdSe}: \alpha=2.5-3.4 \times 10^{-4} \mathrm{eV} / \mathrm{K}\right.$ and $\beta=181-315 \mathrm{~K}$; CdTe $\alpha=4.0-5.1 \times 10^{-4} \mathrm{eV} / \mathrm{K}$ and $\beta=102-134 \mathrm{~K}$; (fitting parameters and error SI, S15)], shows that the $\alpha$ and $\beta$ values obtained are closer, albeit on the lower side of the range, to that of bulk CdTe. This implies that the bandgap of the CdTe domain is more sensitive to temperature than CdSe. Cryogenic X-ray diffraction measurements (SI, Figure S13) also show that on cooling both the CdSe and CdTe lattice volumes decrease with the latter contracting more. Taking these results together we rationalize the blue shift as follows: on cooling there is a contraction of $\mathrm{CdSe}$ and $\mathrm{CdTe}$ lattices and a raising of their respective bandgaps. This in turn results in a raising of the $\mathrm{CT}$ state energy and shifts the PL to higher photon energies. Because the 
CdTe lattice is more sensitive to temperature the raising of the CdTe band gap has a dominant contribution in raising the $\mathrm{CT}$ recombination energy.

In addition to blue shift, the spectrum in Figure $7 \mathrm{a}$ also narrows from $130 \mathrm{meV}$ to $70 \mathrm{meV}$ when cooled from room temperature to $4 \mathrm{~K}$. This broader linewidth at high temperatures can be a result of both exciton-phonon coupling and impurities. To determine the relative contribution of these effects we fit the following relation, which has been used to describe the relation between PL linewidth and temperature in many inorganic semiconductors ${ }^{62-64}$ :

$$
\Gamma(\mathrm{T})=\Gamma_{0}+\Gamma_{a c} T+\frac{\Gamma_{L O}}{e^{\frac{E_{L O}}{k T}}-1}+\Gamma_{i m p} e^{-\frac{E_{b}}{k T}} \text { (Equation 2) }
$$

The first term in Equation 2, $\Gamma_{0}$, reflects the inhomogeneous broadening and line width at $0 \mathrm{~K}$. The second term, $\Gamma_{\mathrm{ac}}$ is the coupling strength between excitons and acoustic phonons. The third term represents the homogeneous broadening resulting from LO phonon (Fröhlich) scattering between excitons and optical phonons, where $\Gamma_{\mathrm{LO}}$ is the coupling strength and $E_{\mathrm{LO}}$ is the phonon energy. The final term in this equation describe the inhomogeneous broadening caused by impurities with activation energy $E_{\mathrm{b}}$ and broadening contribution $\Gamma_{\mathrm{imp}}$. The model assumes that phonon modes do not change with temperature, and only considers a single phonon mode. The former may not hold for interfacial states because they are relatively soft. However, given that no phase change occurs when NPLs are cooled and only a single mode can be resolved in the fs-TA experiments the model can be considered as good tool to understand exciton-phonon interactions.

The activation energy of the non-radiative decay pathways due to impurities, $E_{\mathrm{b}}$, was determined to be around $\sim 3 \mathrm{meV}$, as discussed earlier. Inserting this value into Equation 2 (fitting parameters SI, S16), and fitting the FWHM as a function of temperature (Figure 7d) gives values of $75.9 \pm 2.1 \mathrm{meV}\left(\Gamma_{0}\right), 43.8 \pm 1.9 \mathrm{meV}\left(\Gamma_{\mathrm{LO}}\right), 0.010 \pm 0.003 \mathrm{meV} \mathrm{K}^{-1}\left(\Gamma_{\mathrm{ac}}\right)$, and $14.8 \pm 1.7$ $\mathrm{meV}\left(E_{\mathrm{LO}}\right)$. As shown by the blue curve and green curves in Figure $7 \mathrm{~d}$ neither the impurity contribution or acoustic phonon mode fit well the broadening observed (even at low temperatures). On the other hand, the LO-phonon contribution accurately captures the CT PL broadening. The energy of this phonon mode is effectively identical to that observed in the fs-TA experiments (14.8 
meV versus $15.2 \mathrm{meV}$ ). We hence conclude that it is this same interfacial phonon mode that is responsible for the broadening of the CT emission.

\section{Conclusion}

We have investigated charge-transfer excitons in 2D colloidal CdSe/CdTe heterostructures. Using ultrasensitive scatter-free absorption measurements we have shown that these nanoplatelets have an energetically broad CT transition from the ground state, with an uncharacteristically high oscillator strength. Following photoexcitation of the $\mathrm{CdSe} / \mathrm{CdTe}$ domains, the $\mathrm{CT}$ excitons are generated on two timescales: initially via an ultrafast electron transfer ( $\sim 70 \mathrm{fs})$ and then via diffusion from the respective $\mathrm{CdSe} / \mathrm{CdTe}$ domains ( $\sim 5 \mathrm{ps})$. Interestingly, we find that the delocalized CT excitons generated from the initial electron transfer interact with a phonon mode at $120 \mathrm{~cm}^{-1}$. This mode is only present at the $\mathrm{CdSe} / \mathrm{CdTe}$ interface which we suggest acts to localize CT exctions to the interface, as well as being responsible for CT PL line broadening and nonradiative decay. In addition, we uncover the photoluminescence loss pathways in nanoplatelets. At room temperature incomplete diffusion of domain excitons to the interface and non-radiative decay channels of the CT state limit the CT (and total) PLQY to $50 \%$. On cooling to $4 \mathrm{~K}$, however, the total PLQY reaches close to unity with that of the CT state being $\sim 86 \%$; at cryogenic temperatures, losses occur to an emissive sub-bandgap state. This is highly unusual for a nanocrystal system where typically the lowest exciton state is dark and hence the PLQY drops on cooling.

These results have a number of implications for the engineering of 2D colloidal heterostructures. They suggest that to maximize the room temperature quantum yield the lateral width of the CdSe core and CdTe crown should firstly be minimized such as to limit the distance excitons must diffuse to the interface. This will limit losses on transport to the interface from the exposed domains. Secondly improved engineering of the CT interface is required to minimize non-radiative losses from this state. Our results suggest that exciton-phonon scattering is the dominant mechanism in this process and hence tuning of the interfacial structure could be used to minimize this loss channel. This could be achieved through a gradient or alloyed interface which may be 'softer' and the exciton-phonon coupling reduced. A more systematic approach may be through alteration of the relative lateral widths of core and crown which will result in a change of the 
interfacial strain. This approach has been shown to be recently effective at reducing the LO-phonon scattering in $2 \mathrm{D} \mathrm{MoSe}{ }_{2}$ monolayers ${ }^{65}$, resulting in sharper PL linewidths. In addition to their role on the emission yield and linewidth, the results presented suggest that the interaction between CT excitons and phonons plays a role in exciton localization to the core-crown interface. Future work should aim to better characterize the exact role of this mode e.g. via computational modelling.

For use in LEDs the strong exciton-phonon interactions observed may overall not be advantageous, due to the resulting broadening and asymmetry in PL lineshape introduced. On the other hand, for lasing applications the strong exciton-phonon coupling may be highly beneficial. In polariton lasers, interactions with phonons increase the rate of polariton relaxation, hence allowing lasing with extremely low thresholds ${ }^{66,67}$. Given the possibilities for tuning the emission of nanoplatelets via thickness this could lead to short-pulsed lasers with a range of emission wavelengths. Finally, for application in solar cells NPLs may not be well suited because the main carriers generated are CT excitons as opposed to free charges. But the high PL quantum yield and large Stokes shift between absorption and emission make these materials viable candidates for use in luminescent solar concentrators, where minimizing loss of emission through non-radiative channels or reabsorption is highly desirable ${ }^{68}$.

\section{Methods}

$\underline{\text { Sample preparation }}$

CdSe 4-ML NPLs:

$24 \mathrm{mg}$ Se powder, $340 \mathrm{mg}$ of $\mathrm{Cd}(\mathrm{Myr})_{2}$, and $25 \mathrm{~mL}$ of ODE were mixed in a $50 \mathrm{~mL}$ three-neck flask. The solution was degassed under vacuum for $15 \mathrm{~min}$ at room temperature. Then, under an 
argon flow, the temperature is set to $240{ }^{\circ} \mathrm{C}$. A $110 \mathrm{mg}$ amount of $\mathrm{Cd}(\mathrm{Ac})_{2}$ was added when the color becomes orange $\left(200{ }^{\circ} \mathrm{C}\right.$ approximately). After $10 \mathrm{~min}$ at $240{ }^{\circ} \mathrm{C}$, the reaction is quenched with $500 \mu \mathrm{L}$ of oleic acid and then quickly cooled to room temperature. As obtained, the solution is a mix of dots and platelets. After adding $25 \mathrm{~mL}$ of hexane and $25 \mathrm{~mL}$ of ethanol, the platelets are selectively precipitated by centrifugation at $6000 \mathrm{rpm}$ for $5 \mathrm{~min}$. The orange supernatant is removed, and the precipitate is dispersed in hexane. The amount of hexane is chosen so that 100 $\mu \mathrm{L}$ of the NPLs solution diluted in $3 \mathrm{~mL}$ of hexane has an optical density of 0.6 at $512 \mathrm{~nm}$ in a 1 cm cuvette

CdSe/CdTe Core/Crown Heteronanoplatelets:

$1.5 \mathrm{~mL}$ of the CdSe NPLs solution is centrifuged; the platelets are then dried and dispersed in 2 $\mathrm{mL}$ of ODE. In a $25 \mathrm{~mL}$ three-neck flask, $24 \mathrm{mg}$ of $\mathrm{Cd}(\mathrm{Ac})_{2}$, the NPLs in ODE, and $45 \mu \mathrm{L}$ of oleic acid are mixed and vacuumed at room temperature for $15 \mathrm{~min}$. Under an argon flow the temperature is set to $215^{\circ} \mathrm{C}$. In parallel, a solution of TOPTe $(1 \mathrm{M})$ in ODE is prepared (total anion concentration of $0.025 \mathrm{M}$ ). When the temperature is reached, $1.5 \mathrm{~mL}$ of this second solution is injected at a rate of $4 \mathrm{~mL} / \mathrm{h}$ into the reaction media. When the desired injection volume is reached $(1.5 \mathrm{~mL})$, the mixture is cooled to room temperature and washed once with ethanol. The core/crown CdSe/CdTe NPLs are dispersed in hexane and stored in the dark to prevent them from photodegradation.

\begin{abstract}
Absorption Spectroscopy
Linear absorption spectra of colloidal nanoplatelets solutions, placed in a $1 \mathrm{~mm}$ pathlength cuvette (Hellma), were measured using commercial Perkin-Elmer lambda 750 UV-VIS-NIR set-up equipped with a $10 \mathrm{~cm}$ integrating sphere module attachment. A Xe-lamp was used as an excitation source and all measurements were performed under standard ambient conditions. In order to collect the scattered light, the sample cuvette was placed on the front window of the sphere. The spectra were measured simultaneously with the solvent hexane to correct for its absorption.
\end{abstract}

Temperature-Dependent Absorption 
An Agilent Cary 6000i UV-vis-NIR spectrophotometer with blank substrate correction was used. Spin-coated samples on fused silica substrates were placed in a continuous-flow cryostat (Oxford Instruments Optistat CF-V) under helium atmosphere. We allowed the sample temperature to equilibrate for 30 minutes before taking data.

Photoluminescence Measurement, Quantum Yield Determination and Temperature-Dependent PL For room temperature PL measurements, CW $405 \mathrm{~nm}, 532 \mathrm{~nm}, 633 \mathrm{~nm}$ and $647 \mathrm{~nm}$ diode lasers were used to photoexcite nanocrystal solutions. The samples were sufficiently diluted and placed in $0.2 \mathrm{~mm}$ pathlength cuvette (Starna Scientific) such that the effects of reabsorption could be minimised. Emission was measured using an Andor iDus DU420A Si detector.

Quantum yield measurements at low temperature were carried out on spin coated nanoplatelets films via a referencing method detailed in the literature ${ }^{69}$ and explained further in the Supporting Information (SI, Figure S12). In this case films of N,N'-di(hexylheptyl)-perylene-3,4,9,10tetracarboxyl-diimide (HH-PTCDI) in a polystyrene/toluene gel (100\% quantum yield), was used as the reference ${ }^{70}$.

Nanoplatelet samples were spin coated onto Spectrosil glass and transferred from a $\mathrm{N}_{2}$ glove box to a continuous-flow cryostat (Oxford Instruments Optistat V) under helium atmosphere. PL was measured under an excitation power of $\sim 0.1 \mathrm{~W} / \mathrm{cm}^{2}$. We allowed the sample temperature to equilibrate for $30 \mathrm{~min}$ before taking data. No significant difference was found between the PLQY (and lifetimes) of nanoplatelets in film and solution at room temperature indicating that nanoplatelets in film were sufficiently unaggregated.

\section{Photothermal Deflection Spectroscopy}

Photothermal deflection spectroscopy (PDS) measures the refractive index change due to heat that is caused by nonradiative relaxation when the incoming light is absorbed and can be considered a scatter free technique for measuring absorption capable of measuring 5-6 orders of magnitude weaker absorption than the band-edge absorption ${ }^{71}$. For the measurements, a monochromatic pump light beam produced by a combination of a Light Support MKII $100 \mathrm{~W}$ xenon arc source and a CVI DK240 monochromator is shone on the sample (film on Spectrosil fused silica substrate), 
inclined perpendicular to the plane of the sample, which on absorption produces a thermal gradient near the sample surface via nonradiative relaxation induced heating. This results in a refractive index gradient in the area surrounding the sample surface. This refractive index gradient is further enhanced by immersing the sample in an inert liquid FC-72 Fluorinert (3M Company) that has a high refractive index change per unit change in temperature. A fixed wavelength $\mathrm{CW}$ laser probe beam, produced using a Qioptiq $670 \mathrm{~nm}$ fiber-coupled diode laser with temperature stabilizer for reduced beam pointing noise, is passed through this refractive index gradient, producing a deflection proportional to the absorbed light at that particular wavelength, which is detected by a differentially amplified quadrant photodiode and lock-in amplifier (Stanford Research SR830) combination. Scanning through different wavelengths gives the complete absorption spectra.

\section{Steady-State Raman Spectroscopy}

Raman measurements were conducted by back-scattering (T64000, HORIBA) a CW diode line (532 nm, $\sim 1 \mathrm{~mW}$ and $785 \mathrm{~nm}, \sim 1 \mathrm{~mW}$ ) with a triple stage. Spectra were collected $>80 \mathrm{~cm}^{-1}$ where the CCD detector (HORIBA Synapse Open-Electrode) has a monotonically increasing quantum efficiency of between $0.43-0.50$. Acquisitions employed a 100x optical objective and use minimal laser intensity to avoid sample degradation.

\section{Femtosecond Transient Absorption Spectroscopy}

The fs-TA experiments were performed using an Yb-based amplified system (PHAROS, Light Conversion) providing $14.5 \mathrm{~W}$ at $1030 \mathrm{~nm}$ and $38 \mathrm{kHz}$ repetition rate. The probe beam is generated by focusing a portion of the fundamental in a $4 \mathrm{~mm}$ YAG substrate and spans from 520 to 1400 $\mathrm{nm}$. The pump beam is generated in a home built noncollinear optical parametric (NOPAs; $37^{\circ}$ cut BBO, type I, $5^{\circ}$ external angle) pumped with either the second or third harmonic of the source. The NOPAs output ( $4-5 \mathrm{~mW}$ power) was centered either at $520 \mathrm{~nm}, 660 \mathrm{~nm}$ or $860 \mathrm{~nm}$ and pulses were compressed using a chirped mirror wedge prism (Layterc) combination to a temporal duration of $12 \mathrm{fs}$ and $17 \mathrm{fs}$ respectively (upper limit determined by SHG-FROG). The white light is delayed using a computer-controlled piezoelectric translation stage, and a sequence of probe pulses with and without pump is generated using a chopper wheel on the pump beam. The pump irradiance was at $19 \mu \mathrm{J} / \mathrm{cm}^{2}$. After the sample, the probe pulse is split with a $950 \mathrm{~nm}$ dichroic mirror (Thor labs). The visible light $(520-950 \mathrm{~nm})$ is then imaged with a Silicon photodiode array camera 
(Stresing Entwicklunsbüro; visible monochromator $550 \mathrm{~nm}$ blazed grating) with the near-infrared proportion of the probe seeded to an IR monochromator (1200 nm blazed grating) and imaged using an InGaAs photodiode array camera (Sensors Unlimited). This technique allows simultaneous collection of the entire probe spectrum in a single shot. Off-sets for the differing spectral response of the detectors is accounted for in the post-processing of data.

\section{Transient Photoluminescence Spectroscopy}

For femtosecond transient photoluminescence measurements the transient grating technique was used, which allows ultrafast ( $\sim 150 \mathrm{fs})$ PL collection in the range $450-800 \mathrm{~nm}^{50}$. Briefly, a Ti:sapphire amplifier system (Spectra-Physics Solstice Ace) operating at $1 \mathrm{kHz}$ generating $80 \mathrm{fs}$ $800 \mathrm{~nm}$ pulses was split into the pump and probe beam arms. The pump beam was generated by second harmonic generation in a BBO crystal and focused onto the sample. Photoluminescence was collimated using a silver off-axis parabolic mirror and focused onto the gate medium. About $80 \mu \mathrm{J} /$ pulse of the $800 \mathrm{~nm}$ laser output is used for the gate beams, which is first raised $25 \mathrm{~mm}$ above the plane of the PL to produce a boxcar geometry and split into a pair of gate beams using a 50/50 beam splitter. The gate beams are focused onto the gate medium (fused silica), crossing at an angle of $\sim 5^{\circ}$ and overlapping with the focused PL. The two gate beams interfere and create a transient grating in the gate medium due to a modulation of the refractive index via the optical Kerr effect. Temporal overlap between the two gate beams is achieved via a manual delay stage. The PL is then deflected on the transient grating, causing a spatial separation of the gated signal from the PL background. Two lenses collimate and focus the gated signal onto the spectrometer entrance (Princeton Instruments SP 2150) after long- and short-pass filters remove scattered pump and gate light, respectively. Gated PL spectra are measured using an intensified CCD camera (Princeton Instruments, PIMAX4). The ( $\sim 10 \mathrm{~ns})$ electronic shutter of the intensified CCD camera was used to further suppress long-lived PL background. PL spectra at each gate time delay are acquired from $\sim 10000$ laser shots. The time delay between pump and gate beams is controlled via a motorized optical delay line on the excitation beam path and a LabVIEW data acquisition program.

For nanosecond spectrally-resolved photoluminescence, measurements were made using an intensified CCD camera (ICCD; Andor iStar CCD 334 Si detector). For the ICCD measurements 
the excitation source was the the same as that detailed for the transient grating measurements. The instrument response function for the setup was measured to be $\sim 1.5 \mathrm{~ns}$ by scattering excitation light into the detector from a piece of frosted glass.

\section{Picosecond Transient Absorption:}

A portion of the output of a Ti:Sapphire amplifier system (Spectra-Physics Solstice) operating at $1 \mathrm{kHz}$, was used to pump an optical parametric amplifier (OPA; TOPAS, Light Conversion) to generate the pump pulse at either 530 or $545 \mathrm{~nm}$ (FWHM $5 \mathrm{~nm},<150 \mathrm{fs}$ ). Another portion of the Ti:Sapphire fundamental was used to seed a lab built non-linear optical parametric amplifier (NOPA), generating probe pulses $520-770 \mathrm{~nm}$. An optical filter was used to remove residual 800 $\mathrm{nm}$ fundamental in the probe spectrum. Thin films of dispersed nanoplatelets were measured under vacuum such as to prevent oxidative degradation. A reference pulse that does not interact with the pump was used to reduce noise resulting from laser fluctuations. To ensure constant excitation densities over the probe region on the sample, the pump diameter on the sample was made ca. 5 times bigger than the probe diameter (95 $\mathrm{m}$ FWHM). The probe and reference beams were dispersed in a spectrometer (Andor, Shamrock SR-303i) and detected using a pair of 16-bit 512pixel linear image sensors (Hamamatsu). The probe was delayed using a mechanical delay stage (Newport) and every second pump pulse was omitted using a mechanical chopper. Data acquisition at $1 \mathrm{kHz}$ was enabled by a custom-built board from Stresing Entwicklunsbüro. The differential transmission $(\Delta \mathrm{T} / \mathrm{T})$ was calculated after accumulating and averaging 1000 "pump on" and "pump off' shots for each data point. The excitation fluence was kept sufficiently low $\left(\sim 8-15 \mu \mathrm{j} / \mathrm{cm}^{2}\right)$ such as to avoid nonlinear effects.

\section{Time correlated single photon counting}

To record the time-resolved photoluminescence decay of the samples, time-correlated single photon counting (TCSPC) was performed. Samples were excited with a pulsed laser (PicoQuant LDH400 $40 \mathrm{MHz}$; repetition rate tuned to $500 \mathrm{kHz}$ with frequency divider) at $407 \mathrm{~nm}$ with the resulting photoluminescence decay collected on a $500 \mathrm{~mm}$ focal length spectrograph (Princeton Instruments, SpectraPro2500i) with a cooled CCD camera. The instrument response was determined by scattering excitation light into the detector using a piece of frosted glass; a value of 265 ps was obtained. The excitation fluence for all samples was similar to that used in transient 
absorption measurements and kept sufficiently low $\left(\sim 12 \mu \mathrm{j} / \mathrm{cm}^{2}\right)$ such as to avoid nonlinear effects e.g. from exciton-exciton annihilation.

\section{$\underline{\text { X-Ray diffraction }}$}

XRD was performed using a Bruker X-ray D8 Advance diffractometer with $\mathrm{Cu} \mathrm{K} \alpha 1,2$ radiation $(\lambda$ $=1.541 \AA$ ). Low-temperature measurements were made on cooling between $300-12 \mathrm{~K}$ using an Oxford Cyrosytem PheniX stage. Spectra were collected with an angular range of $10^{\circ}<2 \theta<60^{\circ}$ and $\Delta \theta=0.01431^{\circ}$ over 60 minutes. Measurements were made on drop casted films from the nanoplatelet suspension onto pre-cleaned silicon substrates.

The Bruker Topas software ${ }^{72}$ was used to carry out Le Bail and Rietveld analysis on the measurements ${ }^{73,74}$. Backgrounds were fit with a Chebyshev polynomial function and the peak shape modeled with a pseudo-Voigt function.

\section{$\underline{\text { TEM }}$}

Platelets were dispersed on a TEM copper grid with a holey carbon support film either by spin coating or drop casting in an inert atmosphere from dilute solution. Bright field TEM and STEMHAADF (Scanning Transmission Electron Microscopy - High Angle Annular Dark field) imaging were carried out in a FEI Tecnai Osiris S/TEM operated at $200 \mathrm{kV}$. Elemental maps were acquired and processed with Hyperspy, a python-based open source toolkit for electron microscopy data analysis, applying a Principal Components Analysis to remove noise the spectra before extraction of the elemental maps. A Non-Negative Matrix Factorisation (NMF) algorithm was also employed to identify the components present in the sample.

\section{$\underline{\text { Data Analysis }}$}

Data analysis was carried out with custom codes written using MATLAB and Origin software. The details of specific algorithms used in the analysis are discussed in the Supporting Information. 


\section{Acknowledgements}

We acknowledge financial support from the EPSRC [EP/M005143/1] and Winton Program for the Physics of Sustainability. The work of SI is supported by the program ANR JCJC NannoDoSe. The authors declare that there are no competing financial interests.

\section{Supporting Information}

Supporting Information Available: Characterization of nanoplatelet films, absorption spectra of pure CdSe and CdTe nanoplatelets, Urbach energy calculations, determination of relative oscillator strengths of nanoplatelets domains, application of Marcus theory to CT exciton formation, pump fluence dependence of femtosecond transient absorption measurements and measurements with an off-resonant excitation pulse, transient absorption measurements involving selective pumping of CdSe or CdTe domains, analysis of vibrational spectra, cryogenic X-Ray diffraction, PL-spectra for sub-band gap excitation of nanoplatelets, calculation of LO-phonon energy from cyrogenic photoluminescence measurements and photoluminescence quantum yield calculations as a function of temperature.

This material is available free of charge via the Internet at http://pubs.acs.org. Additional data related to this publication is available at the University of Cambridge data repository at [url to be added in proof]. 


\section{References}

(1) Ithurria, S.; Tessier, M. D.; Mahler, B.; Lobo, R. P. S. M.; Dubertret, B.; Efros, A. L. Nat. Mater. 2011, 10, 936-941.

(2) Naeem, A.; Masia, F.; Christodoulou, S.; Moreels, I.; Borri, P.; Langbein, W. Phys. Rev. B - Condens. Matter Mater. Phys. 2015, 91.

(3) Scott, R.; Kickhöfel, S.; Schoeps, O.; Antanovich, A.; Prudnikau, A.; Chuvilin, A.; Woggon, U.; Artemyev, M.; Achtstein, A. W. Phys. Chem. Chem. Phys. 2016, $183197-$ 3203.

(4) Dang, C.; Lee, J.; Breen, C.; Steckel, J. S.; Coe-Sullivan, S.; Nurmikko, A. Nat. Nanotechnol. 2012, 7, 335-339.

(5) Son, J. S.; Yu, J. H.; Kwon, S. G.; Lee, J.; Joo, J.; Hyeon, T. Adv. Mat. 2011, 11, 3214 3219.

(6) Baghani, E.; Oleary, S. K.; Fedin, I.; Talapin, D. V.; Pelton, M. J. Phys. Chem. Lett. 2015, 6, 1032-1036.

(7) Olutas, M.; Guzelturk, B.; Kelestemur, Y.; Yeltik, A.; Delikanli, S.; Demir, H. V. ACS Nano 2015, 9, 5041-5050.

(8) Ithurria, S.; Bousquet, G.; Dubertret, B. J. Am. Chem. Soc. 2011, 133, 3070-3077.

(9) Gao, Y.; Weidman, M. C.; Tisdale, W. A. Nano Lett. 2017, 17, 3837-3843.

(10) Vitukhnovsky, A. G.; Lebedev, V. S.; Selyukov, A. S.; Vashchenko, A. A.; Vasiliev, R. B.; Sokolikova, M. S. Chem. Phys. Lett. 2015, 619, 185-188.

(11) Chen, Z.; Nadal, B.; Mahler, B.; Aubin, H.; Dubertret, B. Adv. Funct. Mater. 2014, 24, $295-302$.

(12) Sigle, D. O.; Zhang, L.; Ithurria, S.; Dubertret, B.; Baumberg, J. J. J. Phys. Chem. Lett. 2015, 6, 1099-1103.

(13) Robert, C.; Lagarde, D.; Cadiz, F.; Wang, G.; Lassagne, B.; Amand, T.; Balocchi, A.; Renucci, P.; Tongay, S.; Urbaszek, B.; Marie, X. Phys. Rev. B 2016, 93. 
(14) Tessier, M. D.; Javaux, C.; Maksimovic, I.; Loriette, V.; Dubertret, B. ACS Nano 2012, 6, 6751-6758.

(15) Pedetti, S.; Ithurria, S.; Heuclin, H.; Patriarche, G.; Dubertret, B. J. Am. Chem. Soc. 2014, $136,16430-16438$.

(16) Kelestemur, Y.; Olutas, M.; Delikanli, S.; Guzelturk, B.; Akgul, M. Z.; Demir, H. V. J. Phys. Chem. C 2015, 119, 2177-2185.

(17) Antanovich, A. V.; Prudnikau, A. V.; Melnikau, D.; Rakovich, Y. P.; Chuvilin, A.;

Woggon, U.; Achtstein, A. W.; Artemyev, M. V. Nanoscale 2015, 7, 8084-8092.

(18) Li, Q.; Xu, Z.; McBride, J. R.; Lian, T. ACS Nano 2017, 11, 2545-2553.

(19) Li, Q.; Zhou, B.; McBride, J. R.; Lian, T. ACS Energy Lett. 2017, 2, 174-181.

(20) Cassette, E.; Pedetti, S.; Mahler, B.; Ithurria, S.; Dubertret, B.; Scholes, G. D. Phys. Chem. Chem. Phys. 2017, 19, 8373-8379.

(21) Wu, K.; Li, Q.; Jia, Y.; McBride, J. R.; Xie, Z. X.; Lian, T. ACS Nano 2015, 9, 961-968.

(22) Dufour, M.; Steinmetz, V.; Izquierdo, E.; Pons, T.; Lequeux, N.; Lhuillier, E.; Legrand, L.; Chamarro, M.; Barisien, T.; Ithurria, S. J. Phys. Chem. C 2017, 121, 24816 - 24823

(23) Kelestemur, Y.; Guzelturk, B.; Erdem, O.; Olutas, M.; Erdem, T.; Usanmaz, C. F.; Gungor, K.; Demir, H. V. J. Phys. Chem. C 2017, 121, 4650-4658.

(24) Schoenlein, R. W.; Mittleman, D. M.; Shiang, J. J.; Alivisatos, A. P.; Shank, C. V. Phys. Rev. Lett. 1993, 70, 1014-1017.

(25) Krauss, T. D.; Wise, F. W. Phys. Rev. B - Condens. Matter Mater. Phys. 1997, 55, 98609865.

(26) Milliron, D.; Hughes, S. M.; Cui, Y.; Manna, L.; Li, J.; Wang, L. W.; Alivisatos, A. P. Nature 2004, 430, 190-195.

(27) Kim, S.; Fisher, B.; Eisler, H. J.; Bawendi, M. J. Am. Chem. Soc. 2003, 125, 1146611467.

(28) Jones, M.; Kumar, S.; Lo, S. S.; Scholes, G. D. J. Phys. Chem. C 2008, 112, 5423-5431.

(29) Takagahara, T.; Takeda, K. Phys. Rev. B - Condens. Matter Mater. Phys. 1992, 46, $15578-15581$.

(30) Gong, K.; Zeng, Y.; Kelley, D. F. J. Phys. Chem. C 2013, 117, 20268-20279.

(31) Kurik, M. V. Urbach Rule. Phys. Status Solidi A. 1971, 8, 9-45.

(32) Urbach, F. Phys. Rev. 1953, 92, 1324-1324. 
(33) Greeff, C. W.; Glyde, H. R. Anomalous Urbach Tail in GaAs. Phys. Rev. B - Condens. Matter Mater. Phys. 1995, 51, 1778-1783.

(34) Jiang, D. S.; Jung, H.; Ploog, K. J. Appl. Phys. 1988, 64, 1371-1377.

(35) Ikhmayies, S. J.; Ahmad-Bitar, R. N. J. Mater. Res. Technol. 2013, 2, 221-227.

(36) Guyot-Sionnest, P.; Lhuillier, E.; Liu, H. J. Chem. Phys. 2012, 137.

(37) Rakhshani, A. E. J. Phys. Condens. Matter 2000, 12, 4391-4400.

(38) Kronemeijer, A. J.; Pecunia, V.; Venkateshvaran, D.; Nikolka, M.; Sadhanala, A.;

Moriarty, J.; Szumilo, M.; Sirringhaus, H. Adv. Mater. 2014, 26, 728-733.

(39) Kelley, A. M.; Dai, Q.; Jiang, Z. J.; Baker, J. A.; Kelley, D. F. Chem. Phys. 2013, 422, $272-276$.

(40) Schreder, B.; Schmidt, T.; Ptatschek, V.; Spanhel, L.; Materny, A.; Kiefer, W. J. Cryst. Growth 2000, 214, 782-786.

(41) Dzhagan, V.; Valakh, M. Y.; Kolny-Olesiak, J.; Lokteva, I.; Zahn, D. R. T. Appl. Phys. Lett. $2009,94$.

(42) Trallero-Giner, C.; Debernardi, A.; Cardona, M.; Menéndez-Proupín, E. Phys. Rev. B Condens. Matter Mater. Phys. 1998, 57, 4664-4669.

(43) Amirtharaj, P. M.; Pollak, F. H. Appl. Phys. Lett. 1984, 45, 789-791.

(44) Vodop'yanov, L. K.; Vinogradov, V. S.; Mel’Nik, N. N.; Karczewski, G. JETP Lett. 2003, $77,143-145$.

(45) Smith, A. M.; Mohs, A. M.; Nie, S. Nat. Nanotechnol. 2009, 4, 56-63.

(46) Mukherjee, P.; Lim, S. J.; Wrobel, T. P.; Bhargava, R.; Smith, A. M. J. Am. Chem. Soc. 2016, 138, 10887-10896.

(47) Takagahara, T. J. Lumin. 1996, 70, 129-143.

(48) Hannah, D. C.; Dunn, N. J.; Ithurria, S.; Talapin, D. V.; Chen, L. X.; Pelton, M.; Schatz, G. C.; Schaller, R. D. Phys. Rev. Lett. 2011, 107.

(49) Lin, C.; Gong, K.; Kelley, D. F.; Kelley, A. M. ACS Nano 2015, 9, 8131-8141.

(50) Chen, K.; Gallaher, J. K.; Barker, A. J.; Hodgkiss, J. M. J. Phys. Chem. Lett. 2014, 5, $1732-1737$.

(51) Efros, A. L.; Rosen, M.; Kuno, M.; Nirmal, M.; Norris, D.; Bawendi, M. Phys. Rev. B Condens. Matter Mater. Phys. 1996, 54, 4843-4856.

(52) Shornikova, E. V.; Biadala, L.; Yakovlev, D. R.; Feng, D.; Sapega, V. F.; Flipo, N.; 
Golovatenko, A. A.; Semina, M. A.; Rodina, A. V.; Mitioglu, A. A.; Ballottin, M.V.;

Christianen, P. C. M.; Kusrayev, Y. G.; Nasilowski, M.; Dubertret, B.; Bayer, M. Nano Lett. 2018, 18, 373-380.

(53) Shornikova, E.; Biadala, L.; Yakovlev, D.; Sapega, V.; Kusrayev, Y.; Mitioglu, A.; Ballottin, M.; Christianen, P.; Belykh, V.; Kochiev, M.; Sibeldin, N. N.; Golovatenko, A. A.; Rodina, A. V.; Gippius, N. A.; Kuntzmann, A.; Jiang, Y.; Nasilowski, M.; Dubertret, B.; Bayer, M. Nanoscale 2018, 10, 646-656.

(54) Becker, M. A.; Vaxenburg, R.; Nedelcu, G.; Sercel, P. C.; Shabaev, A.; Mehl, M. J.; Michopoulos, J. G.; Lambrakos, S. G.; Bernstein, N.; Lyons, J. L.; Stöferle, T.; Mahrt, R. F.; Kovalenko, M. V.; Norris, D. J.; Rainò G.; Efros, A. Nature 2018, 553, 189-193.

(55) Leroux, M.; Grandjean, N.; Beaumont, B.; Nataf, G.; Semond, F.; Massies, J.; Gibart, P. Phys. Status Solidi Basic Res. 1999, 216, 605-608.

(56) Akasaka, T.; Gotoh, H.; Saito, T.; Makimoto, T. Appl. Phys. Lett. 2004, 85, 3089-3091.

(57) Varshni, Y. P. Physica 1967, 34, 149-154.

(58) Joshi, A.; Narsingi, K. Y.; Manasreh, M. O.; Davis, E. A.; Weaver, B. D. Appl. Phys. Lett. 2006, 89 .

(59) Camassel, J.; Auvergne, D.; Mathieu, H.; Triboulet, R.; Marfaing, Y. Solid State Commun. 1973, 13, 63-68.

(60) Kosyachenko, L. A.; Sklyarchuk, V. M.; Sklyarchuk, Y. F.; Ulyanitsky, K. S. Semicond. Sci. Technol. 1999, 14, 373-377.

(61) Chin, P. T. K.; Donegá, C. D. M.; Van Bavel, S. S.; Meskers, S. C. J.; Sommerdijk, N. A. J. M.; Janssen, R. A. J. J. Am. Chem. Soc. 2007, 129, 14880-14886.

(62) Lee, J.; Koteles, E. S.; Vassell, M. O. Phys. Rev. B 1986, 33, 5512-5516.

(63) Rudin, S.; Reinecke, T. L. Phys. Rev. B 1990, 41, 3017-3027.

(64) Chen, Y.; Kothiyal, G. P.; Singh, J.; Bhattacharya, P. K. Superlattices Microstruct. 1987, $3,657-664$.

(65) Niehues, I.; Schmidt, R.; Drüppel, M.; Marauhn, P.; Christiansen, D.; Selig, M.;

Berghäuser, G.; Wigger, D.; Schneider, R.; Braasch, L.; Koch, R.; Castellanos-Gomez, A.; Kuhn, T.; Knorr, A.; Malic, E.; Rohfling, M.; de Vascocellos, S. M.; Bratschitsch, R. Nano Lett. 2018, 18, 1751-1757.

(66) Orosz, L.; Réveret, F.; Médard, F.; Disseix, P.; Leymarie, J.; Mihailovic, M.; Solnyshkov, 
D.; Malpuech, G.; Zuniga-Pérez, J.; Semond, F.; Bouchoule, B.; Lafosse, X.; Brimont, C.; Guillet, T. Phys. Rev. B - Condens. Matter Mater. Phys. 2012, 85.

(67) Flatten, L. C.; Christodoulou, S.; Patel, R. K.; Buccheri, A.; Coles, D. M.; Reid, B. P. L.; Taylor, R. A.; Moreels, I.; Smith, J. M. Nano Lett. 2016, 16, 7137-7141.

(68) Bomm, J.; Buchtemann, A.; Chatten, A. J.; Bose, R.; Farrell, D. J.; Chan, N. L. A.; Xiao, Y.; Slooff, L. H.; Meyer, T.; Meyer, A.; van Sark, W. G. J. H. M.; Koole, R. Sol. Energy Mater. Sol. Cells 2011, 95, 2087-2094.

(69) Crosby, G. A.; Demas, J. N. J. Phys. Chem. 1971, 75, 991-1024.

(70) Brouwer, A. M.; Prahl, S.; Fischer, M.; Gardens, D.; Ha, M.; Fax, B. Q. U. K.; Violet, C.; Sulfate, Q.; Resch-Genger, U.; Rurack, K. Pure Appl. Chem. 2013, 85, 2005-2026.

(71) Jackson, W. B.; Amer, N. M.; Boccara, A. C.; Fournier, D. Appl. Opt. 1981, $20,1333$.

(72) Coelho, A. A.; Evans, J.; Evans, I.; Kern, A.; Parsons, S. Powder Diffr. 2011, 26, S22S25.

(73) Rietveld, H. M. J. Appl. Crystallogr. 1969, 2, 65-71.

(74) Le Bail, A.; Duroy, H.; Fourquet, J. L. Mater. Res. Bull. 1988, 23, 447-452. 


\section{For Table of Contents Only}

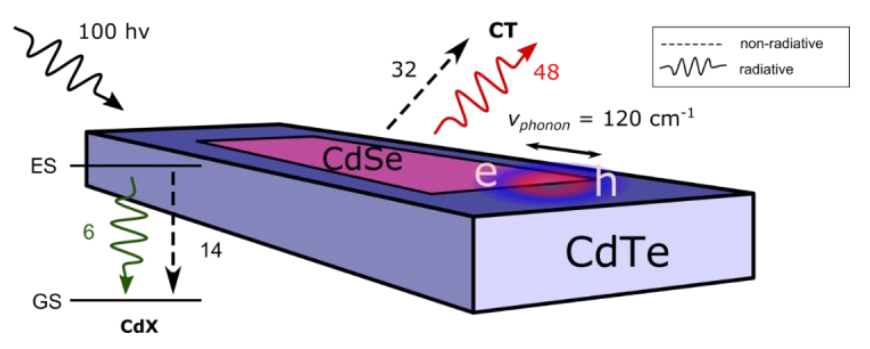

